Review

\title{
Charged Lepton Flavor Violation at the High-Energy Colliders: Neutrino Mass Relevant Particles
}

\author{
Yongchao Zhang
}

check for

updates

Citation: Zhang, Y. Charged Lepton Flavor Violation at the High-Energy Colliders: Neutrino Mass Relevant Particles. Universe 2022, 8, 164. https://doi.org/10.3390/universe 8030164

Academic Editors: Robert H. Bernstein and Bertrand Echenard

Received: 15 December 2021 Accepted: 26 February 2022 Published: 6 March 2022

Publisher's Note: MDPI stays neutral with regard to jurisdictional claims in published maps and institutional affiliations.

Copyright: (C) 2022 by the author Licensee MDPI, Basel, Switzerland. This article is an open access article distributed under the terms and conditions of the Creative Commons Attribution (CC BY) license (https:// creativecommons.org/licenses/by/ $4.0 /)$.
School of Physics, Southeast University, Nanjing 211189, China; zhangyongchao@seu.edu.cn

\begin{abstract}
We summarize the potential charged lepton flavor violation (LFV) from neutrino mass relevant models, for instance the seesaw mechanisms. In particular, we study, in a model-dependent way, the LFV signals at the high-energy hadron and lepton colliders originating from the beyond standard model (BSM) neutral scalar $H$, doubly charged scalar $H^{ \pm \pm}$, heavy neutrino $N$, heavy $W_{R}$ boson, and the $Z^{\prime}$ boson. For the neutral scalar, doubly charged scalar and $Z^{\prime}$ boson, the LFV signals originate from the (effective) LFV couplings of these particles to the charged leptons, while for the heavy neutrino $N$ and $W_{R}$ boson, the LFV effects are from flavor mixing in the neutrino sector. We consider current limits on these BSM particles and estimate their prospects at future high-energy hadron and lepton colliders.
\end{abstract}

Keywords: lepton flavor violation; BSM neutral scalar; doubly charged scalar; heavy neutrino; heavy $W_{R}$ boson; $Z^{\prime}$ boson

\section{Introduction}

In the standard model (SM) of particle physics, charged lepton flavor violation (LFV) processes are vanishing, such as the decays $\ell_{\alpha} \rightarrow \ell_{\beta} \gamma, \ell_{\alpha} \rightarrow \ell_{\beta} \ell_{\gamma} \ell_{\delta}$ (with $\alpha, \beta, \gamma, \delta=e, \mu, \tau$ the lepton flavor indices) and muonium-antimuonium oscillation [1]. Other observables, for instance the anomalous magnetic dipole moments of electron and muon, might also receive sizable contributions from the beyond SM (BSM) particles and their interactions [2]. Therefore, these precision measurements at the high-intensity frontier are one of the primary probes of the BSM physics [3-6]. LFV in the charged lepton sector can be, to some extent, relevant to BSM in the neutrino sector, for instance the various seesaw mechanisms to generate the tiny neutrino masses. Depending on model details and specific UV completions, the BSM particles from the seesaw frameworks can be of spin zero, half, or one, i.e., the CP-even scalars or pseudoscalars, fermions, and vector bosons. In principle, these particles can be either heavy at the TeV scale or relatively light at the GeV scale or even lighter, if allowed by experimental data. Furthermore, some of the LFV signals induced by these particles can also be lepton number violating (LNV) [7,8] and/or lepton flavor universaility violating (LFUV) [9-13], which are closely related to neutrino mass generation and the $B$-anomalies [14], respectively. For simplicity, we will not consider LNV or LFUV, however focus here only on LFV [2,15-18].

In this paper, we summarize the LFV effects from a couple of representative particles in the seesaw mechanisms, i.e., the BSM neutral scalar $H$, doubly charged scalar $H^{ \pm \pm}$, heavy neutrino $N$, heavy $W_{R}$ boson, and the $Z^{\prime}$ boson. It is worked as much in a model independent way as possible, and we do not consider too much dependence on the model details. With given LFV couplings, we will check the relevant LFV limits from current data, and estimate the prospects of these particles at future high-energy lepton and hadron colliders. More details can be found in the original papers.

\section{BSM Neutral Scalar $H$}

If a BSM neutral scalar couples directly to the SM quarks or mixes with the SM Higgs, it may induce flavor-changing neutral currents (FCNCs) in the quark sector, which are 
stringently constrained by the $K$ and $B$ meson data [1]. Therefore, for simplicity, we assume the neutral scalar $H$ does not couple directly to the SM quarks and its mixing with the SM Higgs $h$ is sufficiently small. In a large variety of BSM scenarios, the scalar $H$ can couple to the SM-charged leptons in a flavor-violating way [19-22], ${ }^{1}$ e.g., in the left-right symmetric model (LRSM) [36-39], two Higgs doublet model [40-42] , $R$-parity violating supersymmetric theories [43-46], and mirror models [47-50]. The LFV couplings of $H$ can arise at the tree-level or 1-loop level, depending on model details. The scalar $H$ can be from a BSM singlet, doublet, triplet, or other multiplets, and thus has different quantum numbers (such as the isospin), gauge couplings, which will provide different production channels of $H$ at the high-energy colliders. In this paper, we focus only on the production of $H$ at future lepton colliders via the LFV couplings in a model independent way. In the perspective of effective theory, the couplings of $H$ to the SM-charged leptons can be written in the following way. ${ }^{2}$

$$
\mathcal{L}_{Y}=h_{\alpha \beta} \bar{\ell}_{\alpha} H \ell_{\beta}+\text { H.c. , }
$$

where $\ell$ is the charged lepton, and $h_{\alpha \beta}$ is the Yukawa coupling. For simplicity, we have assumed $H$ to be a real field and CP to be even. As a result, the matrix $h_{\alpha \beta}$ is symmetric.

The couplings $h_{\alpha \beta}$ of the scalar $H$ can induce very rich LFV signals at the high-energy colliders. In light of the clean backgrounds, the future $e^{+} e^{-}$colliders are the primary facilities to search for such smoking-gun signals, such as at the International Linear Collider (ILC) [51], Circular Electron-Positron Collider (CEPC) [52], Future Circular Collider (FCCee) [53], and Compact Linear Collider (CLIC) [54]. Given a single LFV coupling $h_{\alpha \beta}$ with $\alpha \neq \beta$, if kinematically allowed, the scalar $H$ can be on-shell produced at high-energy $e^{+} e^{-}$ colliders via the process:

$$
e^{+} e^{-} \rightarrow \ell_{\alpha}^{ \pm} \ell_{\beta}^{\mp} H
$$

The Feynman diagrams can be found in Figure 1. For simplicity, it is assumed that all other Yukawa couplings in the matrix $h_{\alpha \beta}$ are vanishing. As a result, a single coupling $h_{\alpha \beta}$ can not induce the LFV decays, such as $\ell_{\beta} \rightarrow \ell_{\alpha} \gamma$ and $\ell_{\beta} \rightarrow 3 \ell_{\alpha}$, which depend on the combinations of $h_{\alpha \beta}$ with other Yukawa couplings, e.g., $h_{\alpha \alpha} h_{\alpha \beta}$. It turns out that only a few precise LFV measurements can be used to set limits on the single coupling $h_{\alpha \beta}$.

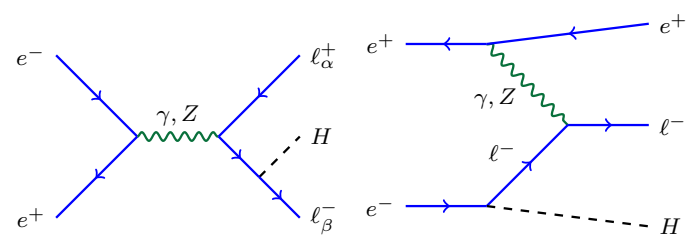

Figure 1. Representative Feynman diagrams for on-shell production of $H$ at future lepton colliders via the process in Equation (2). Figure from Ref. [20].

The coupling $h_{e \mu}$ can induce muonium-antimuonium oscillation at the tree-level, contributes to the anomalous magnetic moment $a_{e}$ of electron via the $H-\mu$ loop, and affects also the measurements of $e^{+} e^{-} \rightarrow \mu^{+} \mu^{-}$at the LEP. The corresponding Feynman diagrams are shown in Figure 2. The precise measurements of muonium oscillation by MACS [55], the current value of $a_{e}$ [56] and the LEP data [57] exclude a large region in the parameter space of the scalar mass $m_{H}$ and the coupling $h_{e \mu}$, as depicted by the shaded regions in Figure 3. More calculation details can be found in Refs. [20,58]. The coupling $h_{e \mu}$ contributes also to the magnetic moment $a_{\mu}$ of muon via the $H-e$ loop. However, this contribution is highly suppressed by the electron mass, and can not explain the current discrepancy of the muon $g-2$ anomaly $\Delta a_{\mu}[59,60]$ (cf. the left panel of Figure 3). 

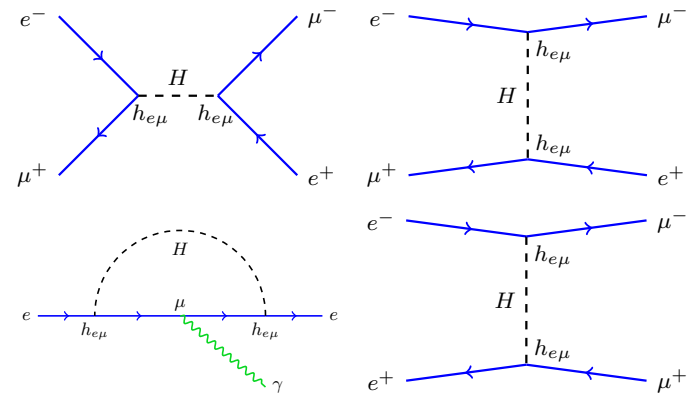

Figure 2. Feynman diagrams for the $H$ contribution to muonium-antimuonium oscillation (upper left and right), electron $g-2$ (lower left), and the LEP $e^{+} e^{-} \rightarrow \mu^{+} \mu^{-}$process (lower right) with the LFV coupling $h_{e \mu}$. Figure from Ref. [20].
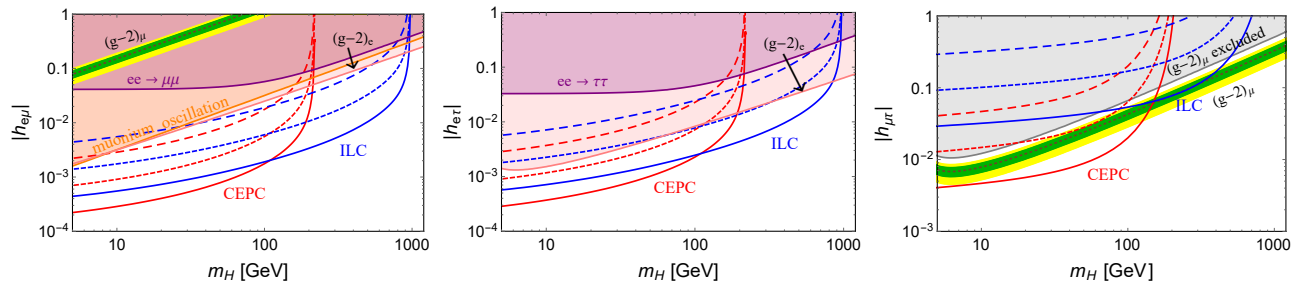

Figure 3. Prospects of LFV couplings $\left|h_{e \mu}\right|$ (left), $\left|h_{e \tau}\right|$ (middle), and $\left|h_{\mu \tau}\right|$ (right) from the process (2) at CEPC $240 \mathrm{GeV}$ with $5 \mathrm{ab}^{-1}$ (red) and ILC $1 \mathrm{TeV}$ with $1 \mathrm{ab}^{-1}$ (blue). A branching ratio of $1 \%$ (long-dashed), $10 \%$ (short-dashed), or $100 \%$ (solid) from $H$ decay is assumed to be visible. The shaded regions are excluded by the corresponding limits. In the left and right panels, the brown line could fit the central value of $\Delta a_{\mu}$, and the green and yellow bands cover the $1 \sigma$ and $2 \sigma$ ranges of $\Delta a_{\mu}$. See text for more details. Figure from Ref. [20].

The coupling $h_{e \mu}$ can induce the signal $e^{+} e^{-} \rightarrow e^{ \pm} \mu^{\mp} H$ at future high-energy $e^{+} e^{-}$ colliders. After being produced, the scalar $H$ can decay back into a pair of opposite-sign different flavor-charged leptons, i.e., $H \rightarrow e^{ \pm} \mu^{\mp}$. In the SM, such processes are absent, and the most important backgrounds at $e^{+} e^{-}$colliders are mainly from electrons mis-identified as muons or vice versa. Therefore, the LFV signal of $e \mu$ resonance can be easily distinguished from the SM backgrounds. Taking CEPC $240 \mathrm{GeV}$ with an integrated luminosity of $5 \mathrm{ab}^{-1}$ and ILC $1 \mathrm{TeV}$ with $1 \mathrm{ab}^{-1}$ as benchmark facilities, the resultant prospects of $m_{H}$ and $h_{e \mu}$ are shown as the red and blue lines in the left panel of Figure 3, assuming at least 10 signal events. The long-dashed, short-dashed, and solid lines correspond respectively to the branching ratio (BR) of $H \rightarrow e^{ \pm} \mu^{\mp}$ to be $1 \%, 10 \%$, and $100 \%$. Benefiting from the larger luminosity, the CEPC can probe a smaller $h_{e \mu}$ than ILC, down to $\mathcal{O}\left(10^{-4}\right)$ in the limit of massless $H$. On the other end, when $H$ is heavy, ILC can probe a heavier $H$ than CEPC due to the kinematics reason.

If the LFV coupling, $h_{e \tau}$ is present, it will contribute to the electron $g-2$ via the $H-\tau$ loop and the $e^{+} e^{-} \rightarrow \tau^{+} \tau^{-}$process at LEP. The corresponding constraints are shown as shaded regions in the middle panel of Figure 3 . With the coupling $h_{\mu \tau}$, the neutral scalar $H$ provides a simple explanation for the muon $g-2$ anomaly via the $H-\tau$ loop. The corresponding $1 \sigma$ and $2 \sigma$ ranges of $\Delta a_{\mu}$ in the parameter space of $m_{H}$ and $h_{\mu \tau}$ are presented in the right panel of Figure 3, respectively, as the green and yellow bands, while the gray shaded region is excluded by the muon $g-2$ discrepancy at the $5 \sigma$ C.L. As for the case of $h_{e \mu}$, the prospects of $h_{e \tau}$ and $h_{\mu \tau}$ are shown respectively in the middle and right panels of Figure 3, with again at least 10 signal events. The reconstruction efficiency of $\tau$ lepton has taken conservatively to be $60 \%$ at both CEPC and ILC [51]. For all the three LFV couplings $h_{e \mu, e \tau, \mu \tau}$, even if all the current stringent lepton flavor constraints are taken into account, there are yet large regions of parameter space of $m_{H}$ and $h_{\alpha \beta}$ (with $\alpha \neq \beta$ ) that can be probed at future high-energy $e^{+} e^{-}$colliders, as seen in Figure 3. 
If the couplings $h_{e e}$ and $h_{e \mu}$ are both nonzero, as an s-channel mediator the scalar $H$ will induce the process:

$$
e^{+} e^{-} \rightarrow e^{ \pm} \mu^{\mp}
$$

at high-energy colliders, which is also apparently LFV. The Feynman diagram is shown in the left panel of Figure 4 (with $\ell_{\alpha} \ell_{\beta}=e \mu$ ). However, the coupling $h_{e e} h_{e \mu}$ is severely constrained by the limit from $\mu \rightarrow$ eee [61], which precludes all the prospects of $e^{ \pm} \mu^{\mp}$ at future high-energy lepton colliders. In the $\tau$ sector, the limits are much weaker. Let us consider the process $e^{+} e^{-} \rightarrow e^{+} \tau^{-}$, which can be induced by an s-channel $H$ mediator and the combination $h_{e e}^{+} h_{e \tau}$ of Yukawa couplings (cf. the left panel of Figure 4 for the Feynman diagram). Such couplings are subject to the limits from the decays $\tau \rightarrow e e e, \tau \rightarrow e \gamma$, electron $g-2$, and the LEP data $e^{+} e^{-} \rightarrow e^{+} e^{-}, \tau^{+} \tau^{-}$(the corresponding Feynman diagrams are similar to those in Figure 2), which are shown as the shaded regions in the left panel of Figure 5. Requiring at least 10 signal events, the prospects of $m_{H}$ and $\left|h_{e e}^{\dagger} h_{e \tau}\right|$ at CEPC $240 \mathrm{GeV}$ and ILC $1 \mathrm{TeV}$ are presented in the left panel of Figure 5, respectively, as the solid and blue lines. The dips are due to the resonant production of $H$ at CEPC and ILC with $m_{H} \simeq \sqrt{s}$. As $H$ is only in the mediator for the LFV processes, its mass can go well above the center-of-mass energy $\sqrt{s}$ at the lepton colliders, as seen in the three panels of Figure 5 . In the limit of $m_{H} \gg \sqrt{s}$, the high-energy lepton colliders probe the effective four-fermion interaction $(\bar{e} e)(\bar{e} \tau) / \Lambda^{2}$ with the cut-off scale $\Lambda \simeq m_{H} / \sqrt{\left|h_{e e}^{\dagger} h_{e \tau}\right|}[62-67]$.

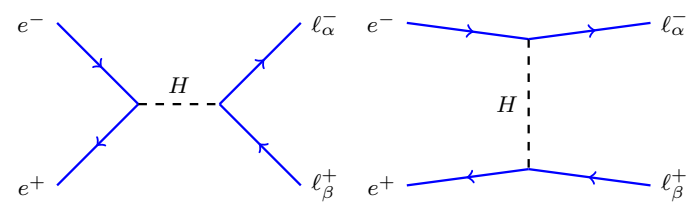

Figure 4. Feynman diagrams for off-shell production of $H$ at future lepton colliders (cf. the process in Equation (3)). Figure from Ref. [20].
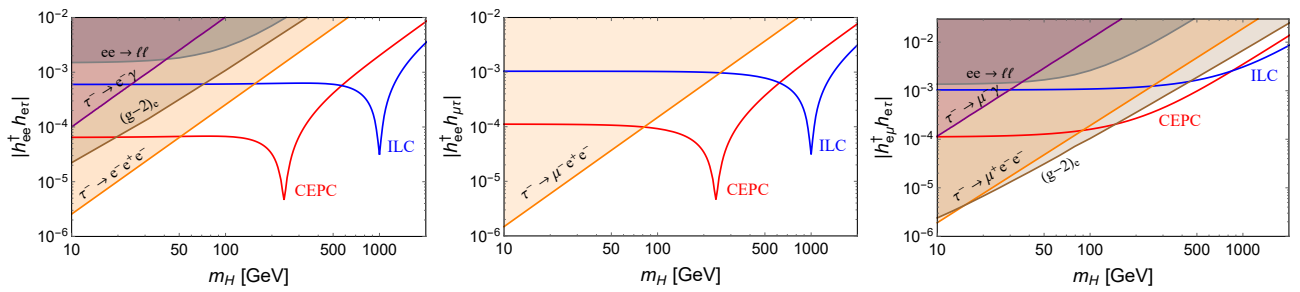

Figure 5. Prospects of $\left|h_{e e}^{\dagger} h_{e \tau}\right|$ (left), $\left|h_{e e}^{\dagger} h_{\mu \tau}\right|$ (middle), and $\left|h_{e \mu}^{\dagger} h_{e \tau}\right|$ (right) from searches of $e^{+} e^{-} \rightarrow$ $e^{ \pm} \tau^{\mp}, \mu^{ \pm} \tau^{\mp}$ at CEPC $240 \mathrm{GeV}$ with $5 \mathrm{ab}^{-1}$ (red) and ILC $1 \mathrm{TeV}$ with $1 \mathrm{ab}^{-1}$ (blue). The shaded regions are excluded by the corresponding limits. See text for more details. Figure from Ref. [20].

To obtain the signatures of $e^{+} e^{-} \rightarrow \mu^{+} \tau^{-}$, there are two different combinations of Yukawa couplings, i.e., $h_{e e}^{\dagger} h_{\mu \tau}$ and $h_{e \mu}^{\dagger} h_{e \tau}$, which are mediated respectively by an $s-$ and $t$-channel $H$ (cf. the Feynman diagrams in the left and right panels of Figure 4). For the case of $h_{e e}^{+} h_{\mu \tau}$, there is only constraint from the decay $\tau^{-} \rightarrow \mu^{-} e^{+} e^{-}$[68], while for $h_{e \mu}^{+} h_{e \tau}$ the limits are mainly from the rare decays $\tau^{-} \rightarrow \mu^{+} e^{-} e^{-}, \tau^{-} \rightarrow \mu^{-} \gamma$, electron $g-2$, and the LEP data $e^{+} e^{-} \rightarrow \mu^{+} \mu^{-}, \tau^{+} \tau^{-}$. These constraints are shown respectively in the middle and right panels of Figure 5 as the shaded regions. The prospects of $h_{e e}^{\dagger} h_{\mu \tau}$ and $h_{e \mu}^{\dagger} h_{e \tau}$ at the CEPC and ILC are also shown in these two figures as the red and blue lines. As the coupling combination $h_{e \mu}^{\dagger} h_{e \tau}$ can not induce any s-channel process at colliders, the prospects in the right panel of Figure 5 do not have any resonance structure. For all these $H$-induced LFV processes $e^{+} e^{-} \rightarrow e^{ \pm} \tau^{\mp}, \mu^{ \pm} \tau^{\mp}$, the future high-energy $e^{+} e^{-}$colliders can probe a large unconstrained parameter space of $m_{H}$ and $h_{\alpha \beta}^{+} h_{\delta \gamma}$, as seen in Figure 5.

Before switching to the doubly-charged scalar in the next section, here are a few more comments on $H$ : 
- $\quad$ Analogous to the neutral current process in Equation (2), we can also have charged current process $e^{+} e^{-} \rightarrow \bar{v}_{\alpha} v_{\beta} H$, which is mediated by a $t$-channel $W$ boson. See Ref. [58] for more details.

- $\quad$ At future high-energy $e^{+} e^{-}$colliders, high-energy photon beams can be obtained from the back scattering of high-intensity low-energy laser beam off high-energy electron beams [69-71], which provides more production channels for the neutral scalar $H$, e.g., via the process $\gamma \gamma \rightarrow e^{ \pm} \mu^{\mp} H$ (the corresponding Feynman diagram is similar to the third diagram in Figure 6). These channels are largely complementary to the $e^{+} e^{-}$ channels above [58].

- $\quad$ Future high-energy muon colliders can probe larger regions of parameter space for the muon flavor couplings, in particular for the explanation of muon $g-2$ anomaly (see e.g., Ref. [72]).

- The LFV signal due to the scalar $H$ can also be searched for at the hadron colliders, i.e., in the channel $q \bar{q} \rightarrow \ell_{\alpha}^{ \pm} \ell_{\beta}^{\mp} H$ at the parton level. As a result of the relatively "dirty" backgrounds at the hadron colliders in particular for the tau leptons, the searches of LFV signals from $H$ is more challenging at the hadron colliders than at the lepton colliders. However, the scalar $H$ can be probed to a larger mass at the hadron colliders (cf. Ref. [73]).
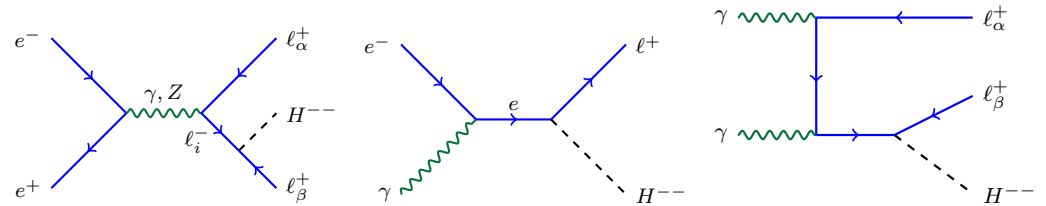

Figure 6. Representative Feynman diagrams for on-shell production of $H^{ \pm \pm}$at future lepton colliders (cf. the processes in Equation (5)). Figure from Ref. [58].

\section{Doubly Charged Scalar $\boldsymbol{H}^{ \pm \pm}$}

The doubly charged scalar $H^{ \pm \pm}$exists in a large variety of BSM scenarios, such as the type-II seesaw [74-78], LRSM [79-81], and the Zee-Babu model [82]. The doubly charged scalars can couple either to left-handed or right-handed charged fermions in the SM, and the Yukawa couplings can be written in the form of, from the perspective of effective Lagrangian: ${ }^{3}$

$$
\mathcal{L}_{Y}=f_{\alpha \beta} H^{++} \overline{\ell_{\alpha}^{C}} \ell_{\beta}+\text { H.c. }
$$

which is not only LNV if the doubly charged scalar does not carry any lepton number [83], ${ }^{4}$ but also potentially LFV. As a result of the gauge couplings of $H^{ \pm \pm}$to photon as well as the $Z$ boson and the Yukawa couplings $f_{\alpha \beta}, H^{ \pm \pm}$can be pair produced at the high-energy lepton and hadron colliders, i.e., $e^{+} e^{-}, p p \rightarrow H^{++} H^{--}$. The usual gauge interaction inducing the Drell-Yan process can not be used to directly probe the Yukawa couplings $f_{\alpha \beta}$, unless the Yukawa couplings are sufficiently small such that $H^{ \pm \pm}$is long-lived at the high-energy colliders. After being produced, the doubly charged scalars decay into pairs of same-sign dileptons, i.e., $H^{ \pm \pm} \rightarrow \ell_{\alpha}^{ \pm} \ell_{\beta}^{ \pm}$, which is apparently signatures beyond the SM. In some models, the doubly charged scalar may decay also into other particles, e.g., $H^{ \pm \pm} \rightarrow$ $W^{ \pm} W^{ \pm}$in the type-II seesaw. The most stringent limit on the doubly charged scalar mass $M_{ \pm \pm}$is from the direct searches of same-sign dilepton pairs from $H^{ \pm \pm}$decay at the LHC [84-87] (see e.g., Refs. [88-90] for classic phenomenological studies and Refs. [91-96] for projections of $H^{ \pm \pm}$at future hadron colliders). At the low-energy high-precision frontier, the coupling $f_{\alpha \beta}$ is also constrained by the LFV decays $\ell_{\alpha} \rightarrow \ell_{\beta} \gamma, \ell_{\alpha} \rightarrow \ell_{\beta} \ell_{\gamma} \ell_{\delta}$, the electron and muon $g-2$, muonium oscillation, and the LEP $e^{+} e^{-} \rightarrow \ell^{+} \ell^{-}$data (see e.g., Ref. [58]).

As for the neutral scalar $H$ case above, the doubly charged scalar $H^{ \pm \pm}$can also be singly produced at the high-energy lepton colliders via the Yukawa couplings $f_{\alpha \beta}$ [88,97-101], e.g., $e^{+} e^{-} \rightarrow H^{ \pm \pm} \ell_{\alpha}^{\mp} \ell_{\beta}^{\mp}$ (cf. the left panel of Figure 6). The corresponding production cross section of this channel is proportional to the Yukawa coupling $\left|f_{\alpha \beta}\right|^{2}$, therefore the Yukawa 
coupling $f_{\alpha \beta}$ can be directly measured in such processes. Take $f_{e \mu}$ as an explicit example, with the $e^{ \pm}$beams and the the high-energy photon beams, we can have the following single production processes:

$$
e^{+} e^{-}, \gamma \gamma \rightarrow H^{ \pm \pm} e^{\mp} \mu^{\mp}, \quad e^{ \pm} \gamma \rightarrow H^{ \pm \pm} \mu^{\mp} .
$$

The corresponding Feynman diagrams can be found in Figure 6. For simplicity, we assume the doubly charged scalar decays predominately into $e^{ \pm} \mu^{ \pm}, 5$ which can be ensured if the coupling $f_{e \mu}$ is much larger than other Yukawa couplings and other decay channels of the doubly charged scalar, such as $H^{ \pm \pm} \rightarrow W^{ \pm} W^{ \pm}$are subdominant. With $\operatorname{BR}\left(H^{ \pm \pm} \rightarrow e^{ \pm} \mu^{ \pm}\right)=100 \%$, the corresponding LHC limit on $M_{ \pm \pm}$is shown as the vertical dashed line in the left panel of Figure $7[84,85]$. The coupling $f_{e \mu}$ will also induce extra contribution to $e^{+} e^{-} \rightarrow \mu^{+} \mu^{-}$at the LEP, and the corresponding limit is presented as the pink shaded region in the left panel of Figure 7. The contribution of doubly charged scalars to the muon $g-2$ is always negative, and therefore can not explain the muon $g-2$ anomaly. Furthermore, the doubly charged scalar contribution to muon $g-2$ is highly suppressed by the charged lepton mass [2], thus not shown in Figure 7.
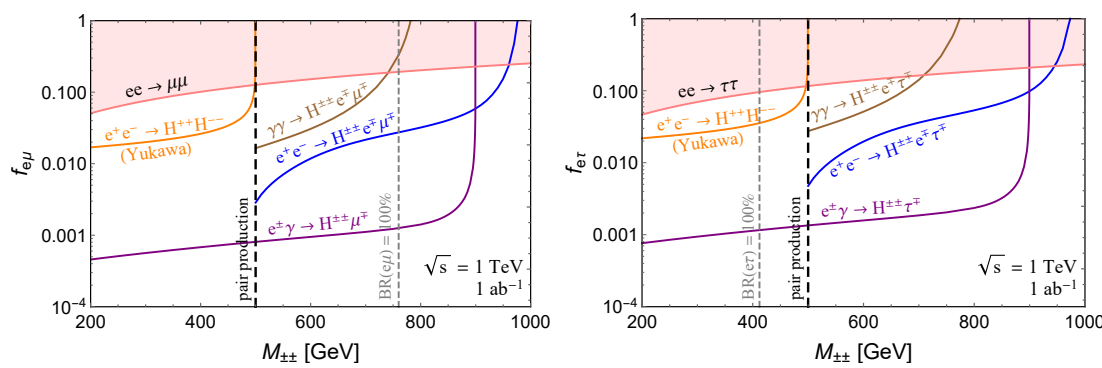

Figure 7. Prospects of the doubly charged scalar mass $M_{ \pm \pm}$and the LFV coupling $f_{e \mu}(\mathbf{l e f t})$ and $f_{e \tau}$ (right) at ILC $1 \mathrm{TeV}$ with $1 \mathrm{ab}^{-1}$, in the Yukawa pair (orange) and single production modes of the $e^{+} e^{-}$(blue), $e \gamma$ (purple) and $\gamma \gamma$ (brown) processes. The pink shaded regions are excluded by the LEP $e^{+} e^{-} \rightarrow \ell^{+} \ell^{-}$data. The vertical dashed gray lines indicate the current same-sign dilepton limits on the doubly charged scalar mass from LHC, assuming respectively, $\mathrm{BR}\left(H^{ \pm \pm} \rightarrow e^{ \pm} \mu^{ \pm}\right)=100 \%$ and $\operatorname{BR}\left(H^{ \pm \pm} \rightarrow e^{ \pm} \tau^{ \pm}\right)=100 \%$ for the left and right panels. See text for more details. Figure from Ref. [58].

The prospects of $H^{ \pm \pm}$and $f_{e \mu}$ at the ILC $1 \mathrm{TeV}$ with luminosity of $1 \mathrm{ab}^{-1}$ are shown in the left panel of Figure 7. For the photon beam, we take the effective photon luminosity distribution from Refs. [69-71]. In the $e^{+} e^{-}$and $\gamma \gamma$ channels, for doubly charged scalar mass $M_{ \pm \pm} \lesssim \sqrt{s} / 2=500 \mathrm{GeV}$, the production of $H^{ \pm \pm}$will be dominated by pair production via both the gauge and Yukawa interactions, while for $M_{ \pm \pm} \gtrsim 500 \mathrm{GeV}$ only the single production of $H^{ \pm \pm}$is kinematically allowed. The $e^{ \pm} \gamma$ process has only two particles in the final state, thus it can probe a smaller Yukawa coupling $f_{e \mu}$. For the LFV coupling $f_{e \tau}$, the prospects at the ILC $1 \mathrm{TeV}$ is to some extent similar to the $f_{e \mu}$ case (cf. the right panel of Figure 7), with a smaller reconstruction efficiency for $\tau$ than muons. For the LFV coupling $f_{\mu \tau}$, the production cross section for $e^{+} e^{-}, \gamma \gamma \rightarrow H^{ \pm \pm} \mu^{\mp} \tau^{\mp}$ is much smaller than that for $H^{ \pm \pm} e^{\mp} \mu^{\mp}$ and $H^{ \pm \pm} e^{\mp} \tau^{\mp}$. As a result, the prospects of $f_{\mu \tau}$ are only at the order of 0.1 [58].

Analogous to the neutral scalar $H$ above, the doubly charged scalar $H^{ \pm \pm}$can also induce LFV processes $e^{+} e^{-} \rightarrow \ell_{\alpha}^{ \pm} \ell_{\beta}^{\mp}$ at the high-energy lepton colliders by playing the role of the $t$-channel mediator (cf. the left panel of Figure 8). The limit from $\mu \rightarrow$ eee is stringent, and it precludes the prospects of $H^{ \pm \pm}$in the channel of $e^{ \pm} \mu^{\mp}$. In the $\tau$ flavor sector, with the coupling $f_{e e}^{\dagger} f_{e \tau}$, the doubly charged scalar can induce the processes (see Figure 8 for diagrams):

$$
e^{+} e^{-} \rightarrow e^{ \pm} \tau^{\mp}, \quad e^{ \pm} \gamma \rightarrow e^{ \pm} e^{ \pm} \tau^{\mp}, \tau^{ \pm} e^{ \pm} e^{\mp}
$$


Similar to the neutral scalar case, the relevant limits for the doubly charged scalar are from the LFV decays $\tau \rightarrow e \gamma, \tau \rightarrow e e e$, and the LEP data $\ell^{+} \ell^{-}$data, which are presented as the shaded regions in the left panel of Figure 9. The prospects of $M_{ \pm \pm}$and $\left|f_{e e}^{\dagger} f_{e \tau}\right|$ at the CEPC $240 \mathrm{GeV}$ with the luminosity of $5 \mathrm{ab}^{-1}$ and ILC $1 \mathrm{TeV}$ with $1 \mathrm{ab}^{-1}$ are shown in the left panel of Figure 9, with red lines for the channel $e^{+} e^{-} \rightarrow e^{ \pm} \tau^{ \pm}$and the blue lines for $e \gamma \rightarrow e e \tau$ (combing the two possible final state in Equation (6)). The dashed lines denote the CEPC sensitivities, and the solid lines are for ILC. As in the neutral scalar case in Section 2, both CEPC and ILC can probe $H^{ \pm \pm}$with a mass larger than the center-of-mass energy in the $e^{+} e^{-}$channel, which corresponds to detecting the effective four-fermion interaction $(\bar{e} e)(\bar{e} \tau)$ with the cut-off scale $\Lambda \simeq M_{ \pm \pm} / \sqrt{\left|f_{e e}^{+} f_{e \tau}\right|}$. With three particles in the final state, the cross section for $e \gamma$ processes are not as competitive as the $e^{+} e^{-}$channel, as seen in the left panel of Figure 9.

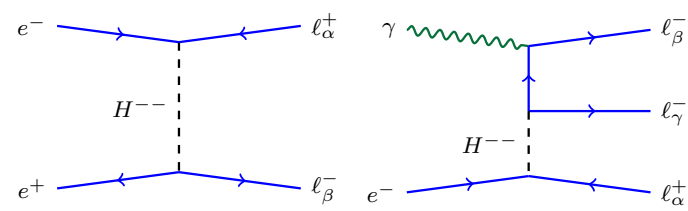

Figure 8. Feynman diagrams for off-shell production of $H^{ \pm \pm}$at future lepton colliders (cf. the processes in Equation (6)). Figure from Ref. [58].
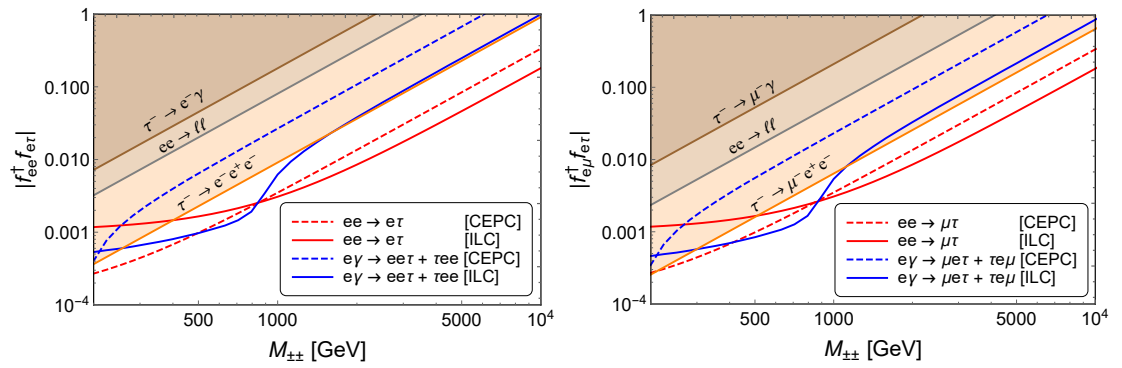

Figure 9. Prospects of the Yukawa couplings $\left|f_{e e}^{\dagger} f_{e \tau}\right|$ (left) and $\left|f_{e \mu}^{\dagger} f_{e \tau}\right|$ (right) for the doubly charged scalar $H^{ \pm \pm}$production via the $e e \rightarrow \ell_{\alpha} \ell_{\beta}$ (red) and $e \gamma \rightarrow \ell_{\alpha} \ell_{\beta} \ell_{\gamma}$ (blue) processes, at CEPC $240 \mathrm{GeV}$ with $5 \mathrm{ab}^{-1}$ (dashed) and ILC $1 \mathrm{TeV}$ with $1 \mathrm{ab}^{-1}$ (solid). The shaded regions are excluded by the corresponding limits. See text for more details. Figure from Ref. [58].

Given the coupling $f_{e \mu}^{+} f_{e \tau}$, the doubly charged scalar $H^{ \pm \pm}$can induce the processes $e^{+} e^{-} \rightarrow \mu^{ \pm} \tau^{\mp}$ and $e^{ \pm} \gamma \rightarrow \mu^{ \pm} e^{ \pm} \tau^{\mp}, \tau^{ \pm} e^{ \pm} \mu^{\mp}$. The corresponding limits from $\tau^{-} \rightarrow \mu^{-} \gamma$, $\tau^{-} \rightarrow \mu^{-} e^{+} e^{-}$, the LEP $\ell^{+} \ell^{-}$data, and prospects of $H^{ \pm \pm}$at the CEPC $240 \mathrm{GeV}$ and ILC 1 $\mathrm{TeV}$ are presented in the right panel of Figure 9. As for the case of $f_{e e}^{+} f_{e \tau}$, the $e^{+} e^{-}$processes have better sensitivities than the $e \gamma$ collisions. These processes can also be induced by the coupling $f_{e e}^{\dagger} f_{\mu \tau}$. However, the corresponding production cross sections are smaller, which weakens the detectability of $f_{e e}^{+} f_{\mu \tau}$ at future high-energy lepton colliders. More $H^{ \pm \pm}$induced LFV processes, such as $e \gamma \rightarrow \mu \mu \mu, \mu \mu \tau$ are also possible, however the corresponding prospects are suppressed by the small cross sections. More details can be found in Ref. [58].

Here are more comments on the doubly charged scalar $H^{ \pm \pm}$:

- $\quad$ The CLIC energy can go up to $3 \mathrm{TeV}$, and this will improve significantly the prospects of $M_{ \pm \pm}$in both the on-shell and off-shell searches at future lepton colliders.

- The future muon collider will provide more channels for searches of LFV due to the doubly charged scalar, for instance in the processes $\mu^{+} \mu^{-} \rightarrow e^{ \pm} \mu^{\mp}, \mu^{ \pm} \tau^{\mp}$. Furthermore, the muon collider can explore higher energy scales than the $e^{+} e^{-}$colliders [102].

- For sufficiently small couplings, the doubly charged scalar can be long-lived at the high-energy colliders, which is however largely model dependent. For instance, in some regions of parameter space in the type-II seesaw, the decays $H^{ \pm \pm} \rightarrow \ell_{\alpha}^{ \pm} \ell_{\beta}^{ \pm}$, $W^{ \pm(*)} W^{ \pm(*)}$ are suppressed, respectively, by the tiny active neutrinos and the small 
vacuum expectation value $v_{\Delta}$ of the triplet (and the off-shell $W$ bosons), which makes $H^{ \pm \pm}$potentially long-lived [90]. The searches of long-lived doubly charged scalar is largely complementary to the searches of prompt signals from $H^{ \pm \pm}$decay [103].

- As mentioned above, the doubly charged scalar can be produced at the high-energy colliders via the gauge interactions, e.g., the Drell-Yan process $p p \rightarrow H^{++} H^{--}$. At future high-energy hadron colliders, the doubly charged scalar can be probed to a higher mass than at the lepton colliders [104]. Though the LFV couplings can not be directly measured in these channels (unless the doubly charged scalar is long-lived at the colliders), the LFV signals at the hadron colliders are largely complementary to the signals in this section at the lepton colliders.

\section{Heavy Neutrino $N$}

In the type-I seesaw [105-109], heavy neutrinos are introduced to generate the tiny neutrino masses. With the mass term $\mathcal{M}_{N, i j} \overline{N_{i}^{C}} N_{j}(i, j$ are the mass indices), the heavy neutrinos $N_{i}$ are Majorana particles. In some other seesaw models such as the inverse seesaw [110-112], by adding three more neutral singlet fermions, the heavy neutrinos form pseudo-Dirac states. Whether they are Majorana or Dirac fermions, the heavy neutrinos could mix with the active neutrinos $v_{\alpha}(\alpha=e, \mu, \tau)$, and thus couple to the SM Higgs, as well as the $W$ and $Z$ bosons through heavy-light neutrino mixing $V_{\alpha N}$. If the heavy neutrinos are at or below the TeV-scale, they can be produced at the high-energy lepton and hadron colliders. For simplicity, let us focus here only on the LFV signals from the heavy neutrinos. To this end, let us neglect the specific UV completions of seesaw models and consider only one heavy neutrino $N=N_{1}$, with the other two states $N_{2,3}$ much heavier and not contributing significantly to the LFV signals.

The heavy neutrino $N$ can be produced at $p p$ colliders via the charged current DrellYan and vector-boson fusion (VBF) process [113-117]: ${ }^{6}$

$$
\begin{aligned}
& q \bar{q}^{\prime} \rightarrow W^{ \pm *} \rightarrow \ell_{\alpha}^{ \pm} N \rightarrow \ell_{\alpha}^{ \pm} \ell_{\beta}{ }^{\mp} W^{ \pm}, \ell_{\alpha}^{ \pm} \ell_{\beta}^{ \pm} W^{\mp}, \\
& W^{ \pm *} \gamma \rightarrow \ell_{\alpha}^{ \pm} N \rightarrow \ell_{\alpha}^{ \pm} \ell_{\beta}{ }^{\mp} W^{ \pm}, \ell_{\alpha}^{ \pm} \ell_{\beta}^{ \pm} W^{\mp} .
\end{aligned}
$$

The representative diagrams are presented in Figure 10, and we have assumed the heavy neutrino mass $m_{N}>m_{W}$ (with $m_{W}$ the $W$ boson mass). If the heavy neutrino $N$ is a Dirac fermion, there will only be opposite-sign charged leptons $\ell_{\alpha}^{ \pm} \ell_{\beta}^{\mp}$ in the final state. For the case of Majorana $N$, there will also be the same-sign charged leptons $\ell_{\alpha}^{ \pm} \ell_{\beta}^{ \pm}$, which is undoubtedly LNV signals beyond the SM. For some mass ranges of $m_{N}$, the gluon-fusion process $g g \rightarrow Z^{*}, h^{*} \rightarrow v N$ is also very important [120-123]. More details about the production channels of $N$ can be found e.g., in Ref. [124]. If the heavy neutrino $N$ mixes with two neutrino flavors $v_{\alpha, \beta}$ (with $\alpha \neq \beta$ ) in the SM, the production and decay of $N$ at the high-energy colliders will produce LFV signals via the charged currents, i.e., $\ell_{\alpha} \neq \ell_{\beta}$ in the final state of the process in Equation (7) [125].
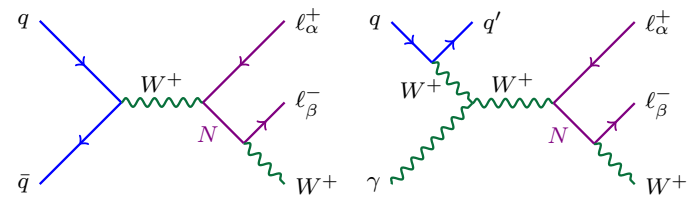

Figure 10. Feynman diagrams for production of heavy neutrino $N$ at future hadron colliders (cf. the processes in Equation (7)). Figure from Ref. [124].

Considering both the charged current Drell-Yan and VBF processes in Equation (7), if the $W$ boson from $N$ decays leptonically, we will have three charged leptons plus significant missing transverse energy (MET) in the final state, with potentially extra (VBF) jets. With $\left|V_{e N}\right|=\left|V_{\mu N}\right|$ and $V_{\tau N}=0$, we can have the processes $p p \rightarrow e e \ell_{X}+e \mu \tau_{h}$, with $\tau_{h}$ referring to the hadronic decaying tauons, $\ell_{X}=e, \mu, \tau_{h}$, and all the possible lepton charges 
are included. Adopting dynamic jet vetoes, the sensitivities of $m_{N}$ and the heavy-light neutrino mixing $\left|V_{e N}\right|=\left|V_{\mu N}\right|$ at future hadron colliders are presented in the left panel of Figure 11 [124]. For the case of $\left|V_{e N}\right|=\left|V_{\tau N}\right|$ with $V_{\mu N}=0$, the charged leptons in the final state can be eee, ee $\mu$, and $e \tau_{h} \ell_{X}$, and the corresponding prospects of $m_{N}$ and $\left|V_{e N}\right|=\left|V_{\tau N}\right|$ are shown in the right panel of Figure 11. It is found that the prospects here are not sensitive to the Majorana or Dirac nature of $N$. In the near future, the LHC $14 \mathrm{TeV}$ data with a luminosity of $300 \mathrm{fb}^{-1}$ can probe heavy-light neutrino mixing angles $\left|V_{e N}\right|^{2}=\left|V_{\mu N}\right|^{2}$ and $\left|V_{e N}\right|^{2}=\left|V_{\tau N}\right|^{2}$ up to the order of $10^{-3}$ for a heavy neutrino at the scale of $100 \mathrm{GeV}$. At future $100 \mathrm{TeV}$ collider, the sensitivity of heavy-light neutrino mixing can even go down to $\mathcal{O}\left(10^{-4}\right)$. More simulation details can be found in Ref. [124]. In Figure 11, the regions above the short dashed lines are excluded by the direct trilepton searches of $N$ at CMS [126], and horizontal dot-dashed lines represent the current electroweak precision data (EWPD) constraints on $\left|V_{\alpha N}\right|$ [127]. More limits on heavy-light neutrino mixing can be found e.g., in Refs. [128-130].
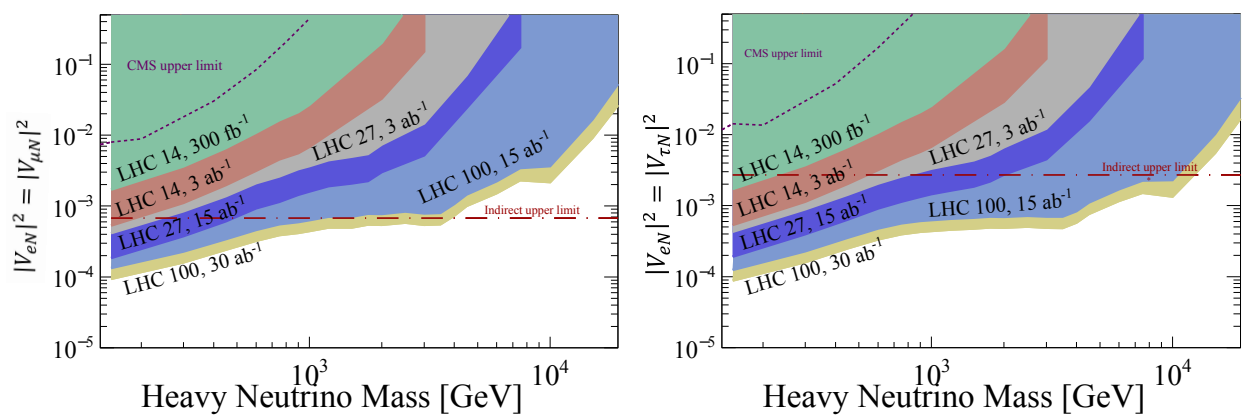

Figure 11. Sensitivities of heavy neutrino mass $m_{N}$ and the heavy-light neutrino mixing $\left|V_{e N}\right|^{2}=\left|V_{\mu N}\right|^{2}$ (left) or $\left|V_{e N}\right|^{2}=\left|V_{\mu N}\right|^{2}$ (right), at future LHC $14 \mathrm{TeV}$ with luminosities of $300 \mathrm{fb}^{-1}$ and $3 \mathrm{ab}^{-1}$, LHC $27 \mathrm{TeV}$ with $3 \mathrm{ab}^{-1}$ and $15 \mathrm{ab}^{-1}$, and $100 \mathrm{TeV}$ collider with $15 \mathrm{ab}^{-1}$ and $30 \mathrm{ab}^{-1}$. Regions above the short dashed lines are excluded by CMS data, and the dot-dashed horizontal lines indicate the indirect limit from current EWPD data. See text for more details. Figure from Ref. [124].

At the GeV-scale, the limits on the neutrino mass $m_{N}$ and $\left|V_{\alpha N}\right|^{2}$ will be mostly from the high-precision LFV measurements below [131-135]:

- At lower energies, the charged current Drell-Yan process in Equation (7) will "hadronize" to be semileptonic weak decays of charged mesons, i.e.,

$$
\mathcal{P}_{2}^{ \pm} \rightarrow \ell_{\alpha} N^{(*)} \rightarrow \ell_{\alpha}^{ \pm} \ell_{\beta}^{\mp} \mathcal{P}_{1}^{ \pm}
$$

with $\mathcal{P}_{1,2}^{ \pm}$the charged mesons. As at the high energy scale, the heavy neutrino $N$ will induce LFV in meson decays if $\ell_{\alpha} \neq \ell_{\beta}$. LNV decays $\mathcal{P}_{2}^{ \pm} \rightarrow \ell_{\alpha}^{ \pm} \ell_{\beta}^{ \pm} \mathcal{P}_{1}^{\mp}$ are also possible if $N$ is a Majorana fermion. It is found that for the mass range of $m_{\pi}<m_{N}<m_{K}$ (with $m_{\pi, K}$ the masses of pions and Kaons), the most stringent limit on $\left|V_{e N} V_{\mu N}\right|$ is from the decay $K^{+} \rightarrow \pi^{+} e^{ \pm} \mu^{\mp}$ leading to $\left|V_{e N} V_{\mu N}\right| \lesssim 10^{-9}$ [136]. For $m_{K}<m_{N}<m_{B}$ (with $m_{B}$ the $B$ meson mass), the strongest limit is from $B^{+} \rightarrow \pi^{+} e^{ \pm} \mu^{\mp}$. In the process (8), if $N$ is on-shell, it can be directly searched via the two-body meson decays, e.g., $K^{+} \rightarrow \ell^{+} N$. The NA62 data have excluded $\left|V_{e N}\right|^{2},\left|V_{\mu N}\right|^{2} \lesssim 10^{-8}$ to $10^{-9}$ for $170 \mathrm{MeV}<m_{N}<450 \mathrm{MeV}$ [137]. More processes can be found e.g., in Refs. [128,133].

- $\quad$ For neutral mesons $\mathcal{P}^{0}$, the heavy neutrino will induce LFV leptonic decays at the 1-loop level, i.e., [138]:

$$
\mathcal{P}^{0} \rightarrow \ell_{\alpha}^{ \pm} \ell_{\beta}^{\mp}
$$

which can be applied to the LFV leptonic decays of $K_{L}, D^{0}, B^{0}$, and $B_{S}^{0}$. Such LFV meson decays are all highly suppressed in the SM, and the mass $m_{N}$ and heavy-light 
neutrino mixing $\left|V_{\alpha N} V_{\beta N}\right|$ are tightly constrained by precision meson data. More details can be found e.g., in Refs. [136,138].

- In the charge lepton sector, if $N$ mixes with two SM neutrino flavors, it will induce extra contribution to the LFV radiative decays of charged leptons $\ell_{\alpha} \rightarrow \ell_{\beta} \gamma$ and $\mu-e$ conversion in nuclei. It is found that the most stringent limit is from $\mu \rightarrow e \gamma$, which leads to $\left|V_{e N} V_{\mu N}\right| \lesssim 10^{-3}$ for a $10 \mathrm{GeV} N$ [128].

- For sufficiently light $N$, it could be long-lived at the high-energy colliders. The corresponding rich phenomenologies can be found e.g., in Refs. [132,133,139-174].

If two (or more) heavy neutrino $N_{1,2}$ are involved, there could be more phenomenological implications, such as heavy neutrino mixing and $\mathrm{CP}$ violation due to the TeV-scale $N$ [175] or in the decays of mesons and the SM Higgs [176-178]. All these processes might involve LFV in some way.

\section{Heavy $W_{R}$ Boson}

In the LRSM, the heavy neutrinos are states from the right-handed isospin doublets, therefore $N$ couples to the heavy right-handed $W_{R}$ boson. As a result of the Majorana nature of $N$, the production and decay of $W_{R}$ at the high-energy hadron colliders will produce same-sign dileptons via the process:

$$
q \bar{q}^{\prime} \rightarrow W_{R}^{ \pm} \rightarrow \ell_{\alpha}^{ \pm} N \rightarrow \ell_{\alpha}^{ \pm} \ell_{\beta}^{ \pm} W_{R}^{\mp *} \rightarrow \ell_{\alpha}^{ \pm} \ell_{\beta}^{ \pm} j j,
$$

which constitutes the smoking-gun signal of LRSM. The Feynman diagram is shown in Figure 12. In such a process, the heavy neutrino mass eigenstate $N$ may be a state of a pure flavor, or combinations of two or more flavors. Take the two flavor case as an explicit example, the heavy neutrino mass eigenstate can be decomposed as $N=\cos \theta N_{\alpha}+\sin \theta N_{\beta}$, with $\theta$ as the mixing angle of heavy neutrinos $N_{\alpha, \beta}$, which leads to LFV signatures $\ell_{\alpha}^{ \pm} \ell_{\beta}^{ \pm}$. The most stringent LHC limit on $W_{R}$ and $N$ is from the recent searches by CMS with a luminosity of $138 \mathrm{fb}^{-1}$ at $13 \mathrm{TeV}$, which covers both the lepton flavor conserving cases $e e$, $\mu \mu$, and LFV case $e \mu$ [179]. No significant excess is found above the backgrounds, and the $W_{R}$ mass is excluded up to $5.4 \mathrm{TeV}$. With $3000 \mathrm{fb}^{-1}$ data at $14 \mathrm{TeV}$, the $W_{R}$ mass can be improved up to roughly $6.5 \mathrm{TeV}[180,181]$. At future $100 \mathrm{TeV}$ colliders, the $W_{R}$ mass can be probed up to roughly $38 \mathrm{TeV}$ with a luminosity of $30 \mathrm{fb}^{-1}$, assuming the heavy neutrino decays promptly [182]. If the neutrino $N$ is relatively light, say at the $100 \mathrm{GeV}$ scale, the parameter space of three-body decay $N \rightarrow \ell j j$ will be highly compressed. The highly compressed signature leads to a boosted topology [182,183], which has been searched at the LHC [184,185]. Projections at future colliders can be found in e.g., Ref. [180]. If sufficiently light, the heavy neutrino $N$ will be long-lived at the high-energy colliders, with the decay length up to the meter level for $m_{N} \sim 10 \mathrm{GeV}[139,180,186-189]$. The displaced vertex signals from $N$ decay can be used to search for the heavy $W_{R}$ boson, and the prospect of $W_{R}$ mass can go up to $33 \mathrm{TeV}$ at the future $100 \mathrm{TeV}$ collider, with $m_{N} \sim 100 \mathrm{GeV}$ [180]. The prompt and displaced vertex signals from $W_{R}$ and $N$ can both be sources for LFV at future high-energy colliders.

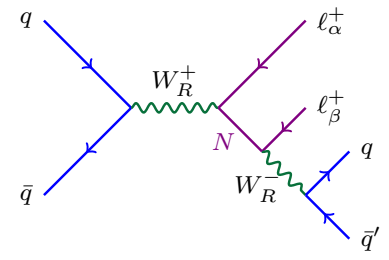

Figure 12. Feynman diagram for production of heavy $W_{R}$ boson at future hadron colliders (cf. the process in Equation (10)). 


\section{6. $Z^{\prime}$ Boson}

The $Z^{\prime}$ gauge boson is associated with a BSM $U(1)^{\prime}$ gauge symmetry. There have been intensive studies on the rich phenomenologies of $Z^{\prime}$, see e.g., Refs. [73,190-199]. The $Z^{\prime}$ boson is intimately related to the neutrino mass generation, e.g., in the $U(1)_{B-L}$ gauge group [196,200-209]. In some cases, the heavy neutral $Z^{\prime}$ boson can have flavor changing neutral currents, ${ }^{7}$ e.g., from the mixing of SM fermions with heavy exotic fermions [214]. Another important motivation of LFV couplings of $Z^{\prime}$ is the muon $g-2$ anomaly, where the $Z^{\prime}$ boson can be either heavy or light. For instance, the effective LFV couplings of $Z^{\prime}$ to $\mu$ and $\tau$ can be written as:

$$
\mathcal{L} \supset Z_{\mu}^{\prime}\left(g_{L}^{\prime} \bar{\mu}_{L} \gamma^{\mu} \tau_{L}+g_{R}^{\prime} \bar{\mu}_{R} \gamma^{\mu} \tau_{R}\right)+\text { H.c. . }
$$

with $\tau$ running in the loop, the $Z^{\prime}$ boson with LFV couplings can explain the muon $g-2$ discrepancy, which is in some sense similar to the neutral scalar case above $[2,215]$. The corresponding $2 \sigma$ regions for the muon $g-2$ anomaly is shown as the green band in Figure 13, where we have assumed that the gauge couplings $g_{L}^{\prime}=g_{R}^{\prime} / 10$.

The direct searches of heavy $Z^{\prime} \rightarrow e \mu, e \tau, \mu \tau$ have been performed at the LHC [216]. Assuming the LFV couplings of $Z^{\prime}$ are the same as the couplings of $Z$ boson to charged leptons, $Z^{\prime}$ mass has been excluded up to $5.0 \mathrm{TeV}$ in the $e \mu$ channel, $4.3 \mathrm{TeV}$ in the $e \tau$ channel, and $4.1 \mathrm{TeV}$ in the $\mu \tau$ channel [216]. The LFV couplings $g_{L, R}^{\prime}$ in Equation (11) are also severely constrained by the decay $\tau \rightarrow \mu Z^{\prime}$ with $Z^{\prime} \rightarrow v_{\mu} \bar{v}_{\tau}$, the lepton flavor universality violation in tauon decays, the high-precision LHC $W$ data, the LEP Z-pole data, and the muon $g-2$ anomaly at the $5 \sigma$ C.L. As seen in Figure 13, when all these constraints are taken into consideration, a $Z^{\prime}$ with mass $m_{Z^{\prime}} \gtrsim 2 \mathrm{GeV}$ can provide viable interpretation of the muon $g-2$ anomaly.

The LFV coupling $g_{L, R}^{\prime}$ of the light $Z^{\prime}$ boson can be directly measured at the highenergy hadron and lepton colliders via the process:

$$
p p, e^{+} e^{-} \rightarrow \mu^{ \pm} \tau^{\mp} Z^{\prime},
$$

which is quite similar to the process (2) for the neutral scalar $H$, and the Feynman diagram is shown in Figure 14 . With $Z^{\prime} \rightarrow \mu^{ \pm} \tau^{\mp}$, the LFV coupling $g_{L, R}^{\prime}$ will generate same-sign dilepton pairs $\mu^{ \pm} \mu^{ \pm} \tau^{\mp} \tau^{\mp}$. The prospects at the high-luminosity LHC (HL-LHC) $14 \mathrm{TeV}$ with an integrated luminosity of $3 \mathrm{ab}^{-1}$ and the FCC-ee $91 \mathrm{GeV}$ with $2.6 \mathrm{ab}^{-1}$ are presented, respectively, as the dotted orange and blue curves in Figure 13. It is clear that both machines can probe large regions of the $Z^{\prime}$ interpretation of the muon $g-2$ anomaly. More details can be found in Ref. [215]. It should be noted that the prospects can be further improved at a future muon collider.

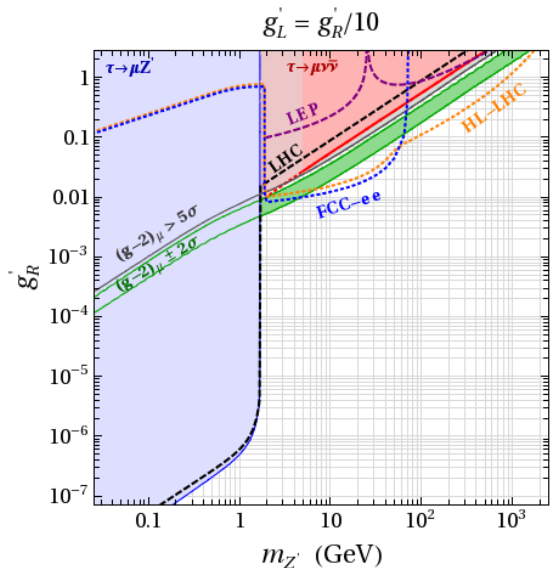

Figure 13. Prospect of $m_{Z^{\prime}}$ and $g_{R}^{\prime}$ at HL-LHC (dotted orange line) and FCC-ee (dotted blue line) with $g_{L}^{\prime}=g_{R}^{\prime} / 10$. The green band corresponds the muon $g-2$ anomaly at the $2 \sigma$ C.L. The blue, gray, 
and red shaded regions are excluded by $\tau \rightarrow \mu Z^{\prime}$, muon $g-2$ discrepancy at the $5 \sigma$ C.L., and $\tau \rightarrow \mu v \bar{v}$. The purple and black dashed lines are, respectively, the limits from the LEP Z-pole data and the LHC W data. See text for more details. Figure from Ref. [215].

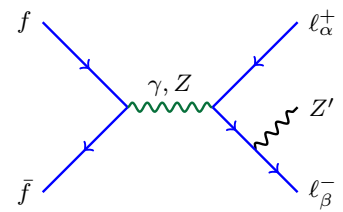

Figure 14. Representative Feynman diagram for on-shell production of $Z^{\prime}$ at future high-energy colliders via the process in Equation (12). Figure from Ref. [215].

With the LFV coupling $g_{e \mu}^{\prime}$ to electron and muon, the $Z^{\prime}$ boson could contribute to $\mu-e$ scattering $[217,218]$, e.g., in the MUonE experiment, which is proposed to determine the contribution of hadronic vacuum polarization to muon $g-2[219,220]$. However, the LFV coupling $g_{e \mu}^{\prime}$ of $Z^{\prime}$ boson is tightly constrained by muonium-antimuonium oscillation and electron $g-2$, which has precluded the sensitivity of the MUonE experiment. More details can be found in Ref. [217].

\section{Conclusions}

In this paper, we summarized briefly the LFV signals from some representative particles in the seesaw frameworks, i.e., the BSM neutral scalar $H$, doubly charged scalar $H^{ \pm \pm}$, heavy neutrino $N$, heavy $W_{R}$ boson, and the $Z^{\prime}$ boson. Constrained by current data, some of these particles are required to be heavy, i.e., the doubly charged scalar and the $W_{R}$ boson, while others can be either heavy at the TeV-scale or light down to the GeV-scale or even lighter, i.e., the scalar $H$, the neutrino $N$ and the $Z^{\prime}$ boson. The LFV signals induced by these particles can originate from the direct (effective) LFV couplings in the charged lepton sector, i.e., the neutral scalar $H$, the doubly charged scalar, and the $Z^{\prime}$ boson. Some of these couplings might be relevant to the neutrino sector, for instance the couplings of doubly charged scalar to charged leptons are largely determined by the neutrino oscillation data in the type-II seesaw. For the heavy $N$ and $W_{R}$ boson, the LFV signals are intimately related to flavor mixing in the neutrino sector, which is transited to the charged lepton sector via the charged currents by the $W$ and $W_{R}$ bosons. This paper focused mainly on the LFV signals induced by these neutrino mass relevant particles at the high-energy lepton and hadron colliders. The LFV signatures can also be searched for at the high-intensity frontier. In particular, if the muon $g-2$ anomaly is revealed to be true, or any LFV signal is found in future experiments, we need to understand better these particles, as well as other well-motivated particles, such as leptoquarks and supersymmetric particles.

Funding: The author is supported by the National Natural Science Foundation of China under grant No. 12175039, the 2021 Jiangsu Shuangchuang (Mass Innovation and Entrepreneurship) Talent Program No. JSSCBS20210144, and the "Fundamental Research Funds for the Central Universities".

Acknowledgments: The author would like to thank the anonymous referees for their valuable comments and suggestions.

Conflicts of Interest: The author declares no conflict of interest.

\section{Notes}

See e.g., Refs. [23-35] for the rich phenomenologies of LFV couplings of the SM Higgs.

Note that without any details on the couplings of $H$ to the active neutrinos the couplings in Equation (1) are not invariant with respect to the $\mathrm{SM} \mathrm{SU}(2)_{L}$ group.

3 As in Equation (1), the couplings in Equation (4) may not be invariant under the SM $S U(2)_{L}$ if the charged leptons involved are left-handed.

4 The lepton number of the doubly charged scalar is model dependent: for instance, $H^{++}$has lepton number $L=-2$ in the type-II seesaw, while in some other models its lepton number can be zero. 
5 In the type-II seesaw, this is not a good approximation, as the leptonic branching fractions are largely determined by the PMNS neutrino mixing matrix, see e.g., Ref. [90].

6 The heavy neutrino $N$ can also be produced from the $W^{+} W^{-}$and $W^{ \pm} W^{ \pm}$scattering processes $[118,119]$.

7 See e.g., Refs. $[23,67,157,190,210-213]$ for the rich phenomenologies of LFV couplings of the SM Z boson.

\section{References}

1. Zyla, P.A.; Barnett, R.M.; Beringer, J.; Dahl, O.; Dwyer, D.A.; Groom, D.E.; Lin, C.-J.; Lugovsky, K.S.; Pianori, E.; Robinson, D.J.; et al. Review of Particle Physics. Prog. Theor. Exp. Phys. 2020, 2020, 083C01. [CrossRef]

2. Lindner, M.; Platscher, M.; Queiroz, F.S. A Call for New Physics: The Muon Anomalous Magnetic Moment and Lepton Flavor Violation. Phys. Rept. 2018, 731, 1-82. [CrossRef]

3. Jaeckel, J.; Ringwald, A. The Low-Energy Frontier of Particle Physics. Ann. Rev. Nucl. Part. Sci. 2010, 60, 405-437. [CrossRef]

4. Hewett, J.L.; Weerts, H.; Brock, R.; Butler, J.N.; Casey, B.C.K.; Collar, J.; de Gouvea, A.; Essig, R.; Grossman, Y.; Haxton, W.; et al. Fundamental Physics at the Intensity Frontier. arXiv 2012, arXiv:1205.2671.

5. $\quad$ Essig, R.; Jaros, J.A.; Wester, W.; Hansson Adrian, P.; Andreas, S.; Averett, T.; Baker, O.; Batell, B.; Battaglieri, M.; Beacham, J.; et al. Working Group Report: New Light Weakly Coupled Particles. arXiv 2013, arXiv:1311.0029.

6. Beacham, J.; Burrage, C.; Curtin, D.; De Roeck, A.; Evans, J.; Feng, J.L.; Gatto, C.; Gninenko, S.; Hartin, A.; Irastorza, I.; et al. Physics Beyond Colliders at CERN: Beyond the Standard Model Working Group Report. J. Phys. G 2020, 47, 010501. [CrossRef]

7. Fileviez Perez, P. New Paradigm for Baryon and Lepton Number Violation. Phys. Rept. 2015, 597, 1-30. [CrossRef]

8. Cai, Y.; Han, T.; Li, T.; Ruiz, R. Lepton Number Violation: Seesaw Models and Their Collider Tests. Front. Phys. 2018, 6, 40. [CrossRef]

9. Algueró, M.; Capdevila, B.; Descotes-Genon, S.; Masjuan, P.; Matias, J. Are we overlooking lepton flavour universal new physics in $b \rightarrow$ sll? Phys. Rev. D 2019, 99, 075017. [CrossRef]

10. Datta, A.; Kumar, J.; London, D. The $B$ anomalies and new physics in $b \rightarrow s e^{+} e^{-}$. Phys. Lett. B 2019, 797, 134858. [CrossRef]

11. Kumar, J.; London, D. New physics in $b \rightarrow s e^{+} e^{-}$? Phys. Rev. D 2019, 99, 073008. [CrossRef]

12. Hurth, T.; Mahmoudi, F.; Santos, D.M.; Neshatpour, S. More indications for lepton nonuniversality in $b \rightarrow s \ell^{+} \ell^{-}$. Phys. Lett. $B$ 2022, 824, 136838. [CrossRef]

13. London, D.; Matias, J. B Flavour Anomalies: 2021 Theoretical Status Report. arXiv 2021, arXiv:2110.13270.

14. Aaij, R.; LHCb Collaboration. Test of lepton universality in beauty-quark decays. arXiv 2021, arXiv:hep-ex/2103.11769.

15. Marciano, W.J.; Mori, T.; Roney, J.M. Charged Lepton Flavor Violation Experiments. Ann. Rev. Nucl. Part. Sci. 2008, 58, 315-341. [CrossRef]

16. Bernstein, R.H.; Cooper, P.S. Charged Lepton Flavor Violation: An Experimenter's Guide. Phys. Rept. 2013, 532, 27-64. [CrossRef]

17. Calibbi, L.; Signorelli, G. Charged Lepton Flavour Violation: An Experimental and Theoretical Introduction. Riv. Nuovo Cim. 2018, 41, 71-174. [CrossRef]

18. Heeck, J. Interpretation of Lepton Flavor Violation. Phys. Rev. D 2017, 95, 015022. [CrossRef]

19. Hou, W.S.; Wong, G.G. $\mu^{+} e^{-} \leftrightarrows \mu^{-} e^{+}$transitions via neutral scalar bosons. Phys. Rev. D 1996, 53, 1537-1541. [CrossRef]

20. Dev, P.S.B.; Mohapatra, R.N.; Zhang, Y. Lepton Flavor Violation Induced by a Neutral Scalar at Future Lepton Colliders. Phys. Rev. Lett. 2018, 120, 221804. [CrossRef]

21. Li, T.; Schmidt, M.A. Sensitivity of future lepton colliders to the search for charged lepton flavor violation. Phys. Rev. D 2019, 99, 055038. [CrossRef]

22. Arganda, E.; Marcano, X.; Mileo, N.I.; Morales, R.A.; Szynkman, A. Model-independent search strategy for the lepton-flavorviolating heavy Higgs boson decay to $\tau \mu$ at the LHC. Eur. Phys. J. C 2019, 79, 738. [CrossRef]

23. Brignole, A.; Rossi, A. Anatomy and phenomenology of $\mu-\tau$ lepton flavor violation in the MSSM. Nucl. Phys. B 2004, 701, 3-53. [CrossRef]

24. Harnik, R.; Kopp, J.; Zupan, J. Flavor Violating Higgs Decays. J. High Energy Phys. 2013, 3, 026. [CrossRef]

25. Blankenburg, G.; Ellis, J.; Isidori, G. Flavour-Changing Decays of a 125 GeV Higgs-like Particle. Phys. Lett. B 2012, 712, 386-390. [CrossRef]

26. Banerjee, S.; Bhattacherjee, B.; Mitra, M.; Spannowsky, M. The Lepton Flavour Violating Higgs Decays at the HL-LHC and the ILC. J. High Energy Phys. 2016, 7, 1-28. [CrossRef]

27. Herrero-Garcia, J.; Rius, N.; Santamaria, A. Higgs lepton flavour violation: UV completions and connection to neutrino masses. J. High Energy Phys. 2016, 11, 1-45. [CrossRef]

28. Chakraborty, I.; Datta, A.; Kundu, A. Lepton flavor violating Higgs boson decay $\boldsymbol{h} \rightarrow \mu \tau$ at the ILC. J. Phys. G 2016, 43,125001 [CrossRef]

29. Chakraborty, I.; Mondal, S.; Mukhopadhyaya, B. Lepton flavor violating Higgs boson decay at $e^{+} e^{-}$colliders. Phy. Rev. D 2017, 96, 115020. [CrossRef]

30. Qin, Q.; Li, Q.; Lü, C.D.; Yu, F.S.; Zhou, S.H. Charged lepton flavor violating Higgs decays at future $e^{+} e^{-}$colliders. Eur. Phys. J. C 2018, 78, 835. [CrossRef]

31. Lu, M.; Levin, A.M.; Li, C.; Agapitos, A.; Li, Q.; Meng, F.; Qian, S.; Xiao, J.; Yang, T. The physics case for an electron-muon collider Adv. High Energy Phys. 2021, 2021, 6693618. [CrossRef] 
32. Arganda, E.; Herrero, M.J.; Marcano, X.; Weiland, C. Imprints of massive inverse seesaw model neutrinos in lepton flavor violating Higgs boson decays. Phys. Rev. D 2015, 91, 015001. [CrossRef]

33. Arganda, E.; Herrero, M.J.; Marcano, X.; Weiland, C. Enhancement of the lepton flavor violating Higgs boson decay rates from SUSY loops in the inverse seesaw model. Phys. Rev. D 2016, 93, 055010. [CrossRef]

34. Arganda, E.; Herrero, M.J.; Marcano, X.; Morales, R.; Szynkman, A. Effective lepton flavor violating $\mathrm{H} \ell \mathrm{i} \ell \mathrm{j}$ vertex from right-handed neutrinos within the mass insertion approximation. Phys. Rev. D 2017, 95, 095029. [CrossRef]

35. Marcano, X.; Morales, R.A. Flavor techniques for LFV processes: Higgs decays in a general seesaw model. Front. Phys. 2020, 7, 228. [CrossRef]

36. Dev, P.S.B.; Mohapatra, R.N.; Zhang, Y. Probing the Higgs Sector of the Minimal Left-Right Symmetric Model at Future Hadron Colliders. J. High Energy Phys. 2016, 5, 174. [CrossRef]

37. Maiezza, A.; Senjanović, G.; Vasquez, J.C. Higgs sector of the minimal left-right symmetric theory. Phys. Rev. D 2017, 95, 095004 [CrossRef]

38. Bhupal Dev, P.S.; Mohapatra, R.N.; Zhang, Y. Displaced photon signal from a possible light scalar in minimal left-right seesaw model. Phys. Rev. D 2017, 95, 115001. [CrossRef]

39. Dev, P.S.B.; Mohapatra, R.N.; Zhang, Y. Long Lived Light Scalars as Probe of Low Scale Seesaw Models. Nucl. Phys. B 2017, 923, 179-221. [CrossRef]

40. Branco, G.C.; Ferreira, P.M.; Lavoura, L.; Rebelo, M.N.; Sher, M.; Silva, J.P. Theory and phenomenology of two-Higgs-doublet models. Phys. Rept. 2012, 516, 1-102. [CrossRef]

41. Crivellin, A.; Kokulu, A.; Greub, C. Flavor-phenomenology of two-Higgs-doublet models with generic Yukawa structure. Phys. Rev. D 2013, 87, 094031. [CrossRef]

42. Crivellin, A.; Heeck, J.; Stoffer, P. A perturbed lepton-specific two-Higgs-doublet model facing experimental hints for physics beyond the Standard Model. Phys. Rev. Lett. 2016, 116, 081801. [CrossRef] [PubMed]

43. Aulakh, C.S.; Mohapatra, R.N. Neutrino as the Supersymmetric Partner of the Majoron. Phys. Lett. B 1982, 119, 136-140. [CrossRef]

44. Hall, L.J.; Suzuki, M. Explicit R-Parity Breaking in Supersymmetric Models. Nucl. Phys. B 1984, 231, 419-444. [CrossRef]

45. Ross, G.G.; Valle, J.W.F. Supersymmetric Models Without R-Parity. Phys. Lett. B 1985, 151, 375-381. [CrossRef]

46. Barbier, R.; Berat, C.; Besancon, M.; Chemtob, M.; Deandrea, A.; Dudas, E.; Fayet, P.; Lavignac, S.; Moreau, G.; Perez, E.; et al. R-parity violating supersymmetry. Phys. Rept. 2005, 420, 1-202. [CrossRef]

47. Hung, P.Q. A Model of electroweak-scale right-handed neutrino mass. Phys. Lett. B 2007, 649, 275-279. [CrossRef]

48. Bu, J.P.; Liao, Y.; Liu, J.Y. Lepton Flavor Violating Muon Decays in a Model of Electroweak-Scale Right-Handed Neutrinos. Phys. Lett. B 2008, 665, 39-43. [CrossRef]

49. Chang, C.F.; Chang, C.H.V.; Nugroho, C.S.; Yuan, T.C. Lepton Flavor Violating Decays of Neutral Higgses in Extended Mirror Fermion Model. Nucl. Phys. B 2016, 910, 293-308. [CrossRef]

50. Hung, P.Q.; Le, T.; Tran, V.Q.; Yuan, T.C. Muon-to-Electron Conversion in Mirror Fermion Model with Electroweak Scale Non-Sterile Right-handed Neutrinos. Nucl. Phys. B 2018, 932, 471-504. [CrossRef]

51. Baer, H.; Barklow, T.; Fujii, K.; Gao, Y.; Hoang, A.; Kanemura, S.; List, J.; Logan, H.E.; Nomerotski, A.; Perelstein, M.; et al. The International Linear Collider Technical Design Report_Volume 2: Physics. arXiv 2013, arXiv:hep-ph/1306.6352.

52. Ahmad, M.; Alves, D.; An, H.; An, Q.; Arhrib, A.; Arkani-Hamed, N.; Ahmed, I.; Bai, Y.; Ferroli, R.B.; Ban, Y.; et al. CEPC-SPPC Preliminary Conceptual Design Report. 1. Physics and Detector. 2015. Available online: http://cepc.ihep.ac.cn/preCDR/main_ preCDR.pdf (accessed on 1 March 2015).

53. Bicer, M.; TLEP Design Study Working Group. First Look at the Physics Case of TLEP. J. High Energy Phys. 2014, 1, 164. [CrossRef]

54. Accomando, E.; CLIC Physics Working Group. Physics at the CLIC multi-TeV linear collider. In Proceedings of the 11th International Conference on Hadron Spectroscopy, Rio de Janeiro, Brazil, 10 June 2005. [CrossRef]

55. Willmann, L.; Schmidt, P.V.; Wirtz, H.P.; Abela, R.; Baranov, V.; Bagaturia, J.; Bertl, W.H.; Engfer, R.; Grossmann, A.; Hughes, V.W.; et al. New bounds from searching for muonium to anti-muonium conversion. Phys. Rev. Lett. 1999, 82, 49-52. [CrossRef]

56. Mohr, P.J.; Newell, D.B.; Taylor, B.N. CODATA Recommended Values of the Fundamental Physical Constants: 2014. Rev. Mod. Phys. 2016, 88, 035009. [CrossRef]

57. Abdallah, J.; DELPHI Collaboration. Measurement and interpretation of fermion-pair production at LEP energies above the $Z$ resonance. Eur. Phys. J. C 2006, 45, 589-632. [CrossRef]

58. Bhupal Dev, P.S.; Mohapatra, R.N.; Zhang, Y. Probing TeV scale origin of neutrino mass at future lepton colliders via neutral and doubly-charged scalars. Phys. Rev. D 2018, 98, 075028. [CrossRef]

59. Bennett, G.W.; Muon g-2 Collaboration. Final Report of the Muon E821 Anomalous Magnetic Moment Measurement at BNL. Phys. Rev. D 2006, 73, 072003. [CrossRef]

60. Abi, B.; Muon g-2 Collaboration. Measurement of the Positive Muon Anomalous Magnetic Moment to 0.46 ppm. Phys. Rev. Lett. 2021, 126, 141801. [CrossRef]

61. Bellgardt, U.; SINDRUM Collaboration. Search for the Decay $\mu^{+} \rightarrow e^{+} e^{+} e^{-}$. Nucl. Phys. B 1988, 299, 1-6. [CrossRef]

62. Kabachenko, V.V.; Pirogov, Y.F. Studying lepton family violation in lepton lepton collisions. Eur. Phys. J. C 1998, 4, 525-532. [CrossRef]

63. Cho, G.C.; Shimo, H. Search for lepton flavor violation at future lepton colliders. Mod. Phys. A 2017, 32, 1750127. [CrossRef] 
64. Ferreira, P.M.; Guedes, R.B.; Santos, R. Lepton flavour violating processes at the International Linear Collider. Phys. Rev. D 2007, 75, 055015. [CrossRef]

65. Aranda, J.I.; Flores-Tlalpa, A.; Ramirez-Zavaleta, F.; Tlachino, F.J.; Toscano, J.J.; Tututi, E.S. Effective Lagrangian description of Higgs mediated flavor violating electromagnetic transitions: Implications on lepton flavor violation. Phys. Rev. D 2009, 79, 093009. [CrossRef]

66. Murakami, B.; Tait, T.M.P. Searching for lepton flavor violation at a future high energy $e^{+} e^{-}$collider. Phys. Rev. D 2015, 91, 015002. [CrossRef]

67. Calibbi, L.; Marcano, X.; Roy, J. Z lepton flavour violation as a probe for new physics at future $e^{+} e^{-}$colliders. Eur. Phys. J. C 2021, 81, 1054. [CrossRef]

68. Hayasaka, K.; Belle collaboration. Search for Lepton Flavor Violating Tau Decays into Three Leptons with 719 Million Produced Tau+Tau- Pairs. Phys. Lett. B 2010, 687, 139-143. [CrossRef]

69. Ginzburg, I.F.; Kotkin, G.L.; Serbo, V.G.; Telnov, V.I. Colliding gamma e and gamma gamma Beams Based on the Single Pass Accelerators (of Vlepp Type). Nucl. Instrum. Meth. 1983, 205, 47-68. [CrossRef]

70. Ginzburg, I.F.; Kotkin, G.L.; Panfil, S.L.; Serbo, V.G.; Telnov, V.I. Colliding gamma e and gamma gamma Beams Based on the Single Pass $e^{+} e^{-}$Accelerators. 2. Polarization Effects. Monochromatization Improvement. Nucl. Instrum. Meth. A 1984, 219, 5-24. [CrossRef]

71. Telnov, V.I. Problems of Obtaining $\gamma \gamma$ and $\gamma \epsilon$ Colliding Beams at Linear Colliders. Nucl. Instrum. Meth. A 1990, $294,72-92$. [CrossRef]

72. Capdevilla, R.; Curtin, D.; Kahn, Y.; Krnjaic, G. Discovering the physics of $(g-2) \mu$ at future muon colliders. Phys. Rev. D 2021, 103, 075028. [CrossRef]

73. Iguro, S.; Mohan, K.A.; Yuan, C.P. Detecting a $\mu \tau$-philic $Z^{\prime}$ boson via photon initiated processes at the LHC. Phys. Rev. D 2020, 101, 075011. [CrossRef]

74. Magg, M.; Wetterich, C. Neutrino Mass Problem and Gauge Hierarchy. Phys. Lett. B 1980, 94, 61-64. [CrossRef]

75. Schechter, J.; Valle, J.W.F. Neutrino Masses in SU(2) $\times$ U(1) Theories. Phys. Rev. D 1980, 22, 2227. [CrossRef]

76. Cheng, T.P.; Li, L.F. Neutrino Masses, Mixings and Oscillations in SU(2) $\times \mathrm{U}(1)$ Models of Electroweak Interactions. Phys. Rev. D 1980, 22, 2860. [CrossRef]

77. Lazarides, G.; Shafi, Q.; Wetterich, C. Proton Lifetime and Fermion Masses in an SO(10) Model. Nucl. Phys. B 1981, 181, 287-300. [CrossRef]

78. Mohapatra, R.N.; Senjanovic, G. Neutrino Masses and Mixings in Gauge Models with Spontaneous Parity Violation. Phys. Rev. D 1981, 23, 165. [CrossRef]

79. Pati, J.C.; Salam, A. Lepton Number as the Fourth Color. Phys. Rev. D 1974, 10, 275-289. Erratum: Phys. Rev. D 1975, 11, 703-703. [CrossRef]

80. Mohapatra, R.N.; Pati, J.C. A Natural Left-Right Symmetry. Phys. Rev. D 1975, 11, 2558. [CrossRef]

81. Senjanovic, G.; Mohapatra, R.N. Exact Left-Right Symmetry and Spontaneous Violation of Parity. Phys. Rev. D 1975, 12, 1502. [CrossRef]

82. Babu, K.S. Model of 'Calculable' Majorana Neutrino Masses. Phys. Lett. B 1988, 203, 132-136. [CrossRef]

83. Crivellin, A.; Ghezzi, M.; Panizzi, L.; Pruna, G.M.; Signer, A. Low- and high-energy phenomenology of a doubly charged scalar. Phys. Rev. D 2019, 99, 035004. [CrossRef]

84. Aaboud, M.; ATLAS Collaboration. Search for doubly charged Higgs boson production in multi-lepton final states with the ATLAS detector using proton-proton collisions at $\sqrt{s}=13$ TeV. Eur. Phys. J. C 2018, 78, 199. [CrossRef] [PubMed]

85. CMS Collaboration. A search for doubly-charged Higgs boson production in three and four lepton final states at $\sqrt{s}=13 \mathrm{TeV}$, CMS-PAS-HIG-16-036. 2017. Available online: http:/ / cds.cern.ch/record/2242956 (accessed on 25 January 2017).

86. Aaboud, M.; ATLAS Collaboration. Search for doubly charged scalar bosons decaying into same-sign $W$ boson pairs with the ATLAS detector. Eur. Phys. J. C 2019, 79, 58. [CrossRef] [PubMed]

87. Aad, G.; ATLAS Collaboration. Search for doubly and singly charged Higgs bosons decaying into vector bosons in multi-lepton final states with the ATLAS detector using proton-proton collisions at $\sqrt{s}=13 \mathrm{TeV}$. J. High Energy Phys. 2021, 6, 146. [CrossRef]

88. Rizzo, T.G. Doubly Charged Higgs Bosons and Lepton Number Violating Processes. Phys. Rev. D 1982, 25, 1355-1364. Addendum: Phys. Rev. D 1983, 27, 657-659. [CrossRef]

89. Akeroyd, A.G.; Aoki, M. Single and pair production of doubly charged Higgs bosons at hadron colliders. Phys. Rev. D 2005, 72, 035011. [CrossRef]

90. Fileviez Perez, P.; Han, T.; Huang, G.Y.; Li, T.; Wang, K. Neutrino Masses and the CERN LHC: Testing Type II Seesaw. Phys. Rev. D 2008, 78, 015018. [CrossRef]

91. Ferreira, M.M.; de Melo, T.B.; Kovalenko, S.; Pinheiro, P.R.D.; Queiroz, F.S. Lepton Flavor Violation and Collider Searches in a Type I + II Seesaw Model. Eur. Phys. J. C 2019, 79, 955. [CrossRef]

92. de Melo, T.B.; Queiroz, F.S.; Villamizar, Y. Doubly Charged Scalar at the High-Luminosity and High-Energy LHC. Int. J. Mod. Phys. A 2019, 34, 1950157. [CrossRef]

93. Padhan, R.; Das, D.; Mitra, M.; Kumar Nayak, A. Probing doubly and singly charged Higgs bosons at the $p p$ collider HE-LHC. Phys. Rev. D 2020, 101, 075050. [CrossRef]

94. Fuks, B.; Nemevšek, M.; Ruiz, R. Doubly Charged Higgs Boson Production at Hadron Colliders. Phys. Rev. D 2020, 101, 075022. [CrossRef] 
95. Gluza, J.; Kordiaczynska, M.; Srivastava, T. Discriminating the HTM and MLRSM models in collider studies via doubly charged Higgs boson pair production and the subsequent leptonic decays. Chin. Phys. C 2021, 45, 073113. [CrossRef]

96. Ashanujjaman, S.; Ghosh, K. Revisiting Type-II see-saw: Present Limits and Future Prospects at LHC. arXiv 2021, arXiv:hep-ph/ 2108.10952.

97. Lusignoli, M.; Petrarca, S. Exotic Higgs Production AT E+ E- Colliders. Phys. Lett. B 1989, 226, 397-400. [CrossRef]

98. Barenboim, G.; Huitu, K.; Maalampi, J.; Raidal, M. Constraints on doubly charged Higgs interactions at linear collider. Phys. Lett. B 1997, 394, 132-138. [CrossRef]

99. Kuze, M.; Sirois, Y. Search for particles and forces beyond the standard model at HERA ep and Tevatron $p \bar{p}$ colliders. Prog. Part. Nucl. Phys. 2003, 50, 1-62. Erratum: Prog. Part. Nucl. Phys. 2004, 53, 583-677. [CrossRef]

100. Yue, C.X.; Zhao, S. Lepton flavor violating signals of a little Higgs model at the high energy linear $e^{+} e^{-}$colliders. Eur. Phys. J. C 2007, 50, 897-903. [CrossRef]

101. Yue, C.X.; Zhao, S.; Ma, W. Single production of the doubly charged scalar in the littlest Higgs model. Nucl. Phys. B 2007, 784, 36-48. [CrossRef]

102. Delahaye, J.P.; Diemoz, M.; Long, K.; Mansoulié, B.; Pastrone, N.; Rivkin, L.; Schulte, D.; Skrinsky, A.; Wulzer, A. Muon Colliders. arXiv 2019, arXiv:physics.acc-ph/1901.06150.

103. Bhupal Dev, P.S.; Zhang, Y. Displaced vertex signatures of doubly charged scalars in the type-II seesaw and its left-right extensions. J. High Energy Phys. 2018, 10, 199. [CrossRef]

104. Arkani-Hamed, N.; Han, T.; Mangano, M.; Wang, L.T. Physics opportunities of a $100 \mathrm{TeV}$ proton-proton collider. Phys. Rept. 2016, 652, 1-49. [CrossRef]

105. Minkowski, P. $\mu \rightarrow e \gamma$ at a Rate of One Out of $10^{9}$ Muon Decays? Phys. Lett. B 1977, 67, 421-428. [CrossRef]

106. Mohapatra, R.N.; Senjanovic, G. Neutrino Mass and Spontaneous Parity Nonconservation. Phys. Rev. Lett. 1980, $44,912$. [CrossRef]

107. Yanagida, T. Horizontal gauge symmetry and masses of neutrinos. Conf. Proc. C 1979, 7902131, 95-99.

108. Gell-Mann, M.; Ramond, P.; Slansky, R. Complex Spinors and Unified Theories. Conf. Proc. C 1979, 790927, $315-321$.

109. Glashow, S.L. The Future of Elementary Particle Physics. NATO Sci. Ser. B 1980, 61, 687. [CrossRef]

110. Mohapatra, R.N. Mechanism for Understanding Small Neutrino Mass in Superstring Theories. Phys. Rev. Lett. 1986, 56, 561-563. [CrossRef]

111. Mohapatra, R.N.; Valle, J.W.F. Neutrino Mass and Baryon Number Nonconservation in Superstring Models. Phys. Rev. D 1986, 34, 1642. [CrossRef]

112. Bernabeu, J.; Santamaria, A.; Vidal, J.; Mendez, A.; Valle, J.W.F. Lepton Flavor Nonconservation at High-Energies in a Superstring Inspired Standard Model. Phys. Lett. B 1987, 187, 303-308. [CrossRef]

113. Keung, W.Y.; Senjanovic, G. Majorana Neutrinos and the Production of the Right-handed Charged Gauge Boson. Phys. Rev. Lett. 1983, 50, 1427. [CrossRef]

114. Datta, A.; Guchait, M.; Pilaftsis, A. Probing lepton number violation via majorana neutrinos at hadron supercolliders. Phys. Rev. D 1994, 50, 3195-3203. [CrossRef] [PubMed]

115. Dev, P.S.B.; Pilaftsis, A.; Yang, U.K. New Production Mechanism for Heavy Neutrinos at the LHC. Phys. Rev. Lett. 2014, 112, 081801. [CrossRef]

116. Alva, D.; Han, T.; Ruiz, R. Heavy Majorana neutrinos from $W \gamma$ fusion at hadron colliders. J. High Energy Phys. 2015, $2,072$. [CrossRef]

117. Degrande, C.; Mattelaer, O.; Ruiz, R.; Turner, J. Fully-Automated Precision Predictions for Heavy Neutrino Production Mechanisms at Hadron Colliders. Phys. Rev. D 2016, 94, 053002. [CrossRef]

118. Dicus, D.A.; Karatas, D.D.; Roy, P. Lepton nonconservation at supercollider energies. Phys. Rev. D 1991, 44, 2033-2037. [CrossRef] [PubMed]

119. Fuks, B.; Neundorf, J.; Peters, K.; Ruiz, R.; Saimpert, M. Majorana neutrinos in same-sign $W^{ \pm} W^{ \pm}$scattering at the LHC: Breaking the TeV barrier. Phys. Rev. D 2021, 103, 055005. [CrossRef]

120. Willenbrock, S.S.D.; Dicus, D.A. Production of Heavy Leptons From Gluon Fusion. Phys. Lett. B 1985, 156, 429-433. [CrossRef]

121. Dicus, D.A.; Roy, P. Supercollider signatures and correlations of heavy neutrinos. Phys. Rev. D 1991, 44, 1593-1596. [CrossRef]

122. Hessler, A.G.; Ibarra, A.; Molinaro, E.; Vogl, S. Impact of the Higgs boson on the production of exotic particles at the LHC. Phys. Rev. D 2015, 91, 115004. [CrossRef]

123. Ruiz, R.; Spannowsky, M.; Waite, P. Heavy neutrinos from gluon fusion. Phys. Rev. D 2017, 96, 055042. [CrossRef]

124. Pascoli, S.; Ruiz, R.; Weiland, C. Heavy neutrinos with dynamic jet vetoes: Multilepton searches at $\sqrt{s}=14,27$, and $100 \mathrm{TeV}$. J. High Energy Phys. 2019, 6, 049. [CrossRef]

125. Arganda, E.; Herrero, M.J.; Marcano, X.; Weiland, C. Exotic $\mu \tau$ jj events from heavy ISS neutrinos at the LHC. Phys. Lett. B 2016, 752, 46-50. [CrossRef]

126. Sirunyan, A.M.; CMS Collaboration. Search for heavy neutral leptons in events with three charged leptons in proton-proton collisions at $\sqrt{s}=13 \mathrm{TeV}$. Phys. Rev. Lett. 2018, 120, 221801. [CrossRef] [PubMed]

127. Fernandez-Martinez, E.; Hernandez-Garcia, J.; Lopez-Pavon, J. Global constraints on heavy neutrino mixing. J. High Energy Phys. 2016, 8, 033. [CrossRef]

128. Bolton, P.D.; Deppisch, F.F.; Bhupal Dev, P.S. Neutrinoless double beta decay versus other probes of heavy sterile neutrinos. J. High Energy Phys. 2020, 3, 170. [CrossRef] 
129. Aad, G.; ATLAS Collaboration. Search for heavy neutral leptons in decays of $W$ bosons produced in $13 \mathrm{TeV} p p$ collisions using prompt and displaced signatures with the ATLAS detector. J. High Energy Phys. 2019, 10, 265. [CrossRef]

130. Tumasyan, A.; CMS Collaboration. Search for long-lived heavy neutral leptons with displaced vertices in proton-proton collisions at $\sqrt{s}=13 \mathrm{TeV}$. arXiv 2022, arXiv:hep-ex/2201.05578.

131. Atre, A.; Han, T.; Pascoli, S.; Zhang, B. The Search for Heavy Majorana Neutrinos. J. High Energy Phys. 2009, 5, 030. [CrossRef]

132. Cottin, G.; Helo, J.C.; Hirsch, M. Displaced vertices as probes of sterile neutrino mixing at the LHC. Phys. Rev. D 2018, $98,035012$. [CrossRef]

133. Coloma, P.; Fernández-Martínez, E.; González-López, M.; Hernández-García, J.; Pavlovic, Z. GeV-scale neutrinos: Interactions with mesons and DUNE sensitivity. Eur. Phys. J. C 2021, 81, 78. [CrossRef]

134. De Vries, J.; Dreiner, H.K.; Günther, J.Y.; Wang, Z.S.; Zhou, G. Long-lived Sterile Neutrinos at the LHC in Effective Field Theory. J. High Energy Phys. 2021, 3, 148. [CrossRef]

135. Zhou, G.; Günther, J.Y.; Wang, Z.S.; de Vries, J.; Dreiner, H.K. Long-lived Sterile Neutrinos at Belle II in Effective Field Theory. arXiv 2021, arXiv:hep-ph/2111.04403.

136. Hu, S.; Wong, S.M.Y.; Xu, F. Probing Sterile Neutrino via Lepton Flavor Violating Decays of Mesons. arXiv 2019, arXiv:hep-ph/ 1904.00568 .

137. Cortina Gil, E.; NA62 Collaboration. Search for heavy neutral lepton production in $K^{+}$decays. Phys. Lett. B 2018, 778, 137-145. [CrossRef]

138. Ilakovac, A. Lepton flavor violation in the standard model extended by heavy singlet Dirac neutrinos. Phys. Rev. D 2000, 62, 036010. [CrossRef]

139. Helo, J.C.; Hirsch, M.; Kovalenko, S. Heavy neutrino searches at the LHC with displaced vertices. Phys. Rev. D 2014, 89, 073005, Erratum: Phys. Rev. D 2016, 93, 099902. [CrossRef]

140. Izaguirre, E.; Shuve, B. Multilepton and Lepton Jet Probes of Sub-Weak-Scale Right-Handed Neutrinos. Phys. Rev. D 2015, 91, 093010. [CrossRef]

141. Dube, S.; Gadkari, D.; Thalapillil, A.M. Lepton-Jets and Low-Mass Sterile Neutrinos at Hadron Colliders. Phys. Rev. D 2017, 96, 055031. [CrossRef]

142. Dib, C.O.; Kim, C.S.; Neill, N.A.; Yuan, X.B. Search for sterile neutrinos decaying into pions at the LHC. Phys. Rev. D 2018, 97, 035022. [CrossRef]

143. Gago, A.M.; Hernández, P.; Jones-Pérez, J.; Losada, M.; Moreno Briceño, A. Probing the Type I Seesaw Mechanism with Displaced Vertices at the LHC. Eur. Phys. J. C 2015, 75, 470. [CrossRef]

144. Accomando, E.; Delle Rose, L.; Moretti, S.; Olaiya, E.; Shepherd-Themistocleous, C.H. Novel SM-like Higgs decay into displaced heavy neutrino pairs in U(1)' models. J. High Energy Phys. 2017, 4, 081. [CrossRef]

145. Caputo, A.; Hernandez, P.; Lopez-Pavon, J.; Salvado, J. The seesaw portal in testable models of neutrino masses. J. High Energy Phys. 2017, 6, 112. [CrossRef]

146. Deppisch, F.F.; Liu, W.; Mitra, M. Long-lived Heavy Neutrinos from Higgs Decays. J. High Energy Phys. 2018, 8, 181. [CrossRef]

147. Liu, J.; Liu, Z.; Wang, L.T. Enhancing Long-Lived Particles Searches at the LHC with Precision Timing Information. Phys. Rev. Lett. 2019, 122, 131801. [CrossRef]

148. Antusch, S.; Cazzato, E.; Fischer, O. Sterile neutrino searches via displaced vertices at LHCb. Phys. Lett. B 2017, 774, 114-118. [CrossRef]

149. Kling, F.; Trojanowski, S. Heavy Neutral Leptons at FASER. Phys. Rev. D 2018, 97, 095016. [CrossRef]

150. Helo, J.C.; Hirsch, M.; Wang, Z.S. Heavy neutral fermions at the high-luminosity LHC. J. High Energy Phys. 2018, 7, 056. [CrossRef]

151. Jana, S.; Okada, N.; Raut, D. Displaced vertex signature of type-I seesaw model. Phys. Rev. D 2018, 98, 035023. [CrossRef]

152. Caputo, A.; Hernandez, P.; Kekic, M.; López-Pavón, J.; Salvado, J. The seesaw path to leptonic CP violation. Eur. Phys. J. C 2017, 77, 258. [CrossRef]

153. Blondel, A.; Graverini, E.; Serra, N.; Shaposhnikov, M. Search for Heavy Right Handed Neutrinos at the FCC-ee. Nucl. Part. Phys. Proc. 2016, 273-275, 1883-1890. [CrossRef]

154. Antusch, S.; Cazzato, E.; Fischer, O. Displaced vertex searches for sterile neutrinos at future lepton colliders. J. High Energy Phys. 2016, 12, 007. [CrossRef]

155. Bonivento, W.; Boyarsky, A.; Dijkstra, H.; Egede, U.; Ferro-Luzzi, M.; Goddard, B.; Golutvin, A.; Gorbunov, D.; Jacobsson, R.; Panman, J.; et al. Proposal to Search for Heavy Neutral Leptons at the SPS. arXiv 2013, arXiv:hep-ex/1310.1762.

156. Abada, A.; Bernal, N.; Losada, M.; Marcano, X. Inclusive Displaced Vertex Searches for Heavy Neutral Leptons at the LHC. J. High Energy Phys. 2019, 1, 093. [CrossRef]

157. Abada, A.; De Romeri, V.; Monteil, S.; Orloff, J.; Teixeira, A.M. Indirect searches for sterile neutrinos at a high-luminosity Z-factory. J. High Energy Phys. 2015, 4, 051. [CrossRef]

158. Antusch, S.; Cazzato, E.; Drewes, M.; Fischer, O.; Garbrecht, B.; Gueter, D.; Klaric, J. Probing Leptogenesis at Future Colliders. J. High Energy Phys. 2018, 9, 124. [CrossRef]

159. Hernández, P.; Jones-Pérez, J.; Suarez-Navarro, O. Majorana vs Pseudo-Dirac Neutrinos at the ILC. Eur. Phys. J. C 2019, 79, 220. [CrossRef]

160. Abada, A.; Arcadi, G.; Domcke, V.; Drewes, M.; Klaric, J.; Lucente, M. Low-scale leptogenesis with three heavy neutrinos. J. High Energy Phys. 2019, 1, 164. [CrossRef] 
161. Boiarska, I.; Bondarenko, K.; Boyarsky, A.; Eijima, S.; Ovchynnikov, M.; Ruchayskiy, O.; Timiryasov, I. Probing baryon asymmetry of the Universe at LHC and SHiP. arXiv 2019, arXiv:hep-ph/1902.04535.

162. Lavignac, S.; Medina, A.D. Displaced Vertex signatures of a pseudo-Goldstone sterile neutrino. J. High Energy Phys. 2021, 1, 151. [CrossRef]

163. Dib, C.O.; Kim, C.S.; Tapia Araya, S. Search for light sterile neutrinos from $W^{ \pm}$decays at the LHC. Phys. Rev. D 2020, 101, 035022 [CrossRef]

164. Drewes, M.; Hajer, J. Heavy Neutrinos in displaced vertex searches at the LHC and HL-LHC. J. High Energy Phys. 2020, 2, 070. [CrossRef]

165. Liu, J.; Liu, Z.; Wang, L.T.; Wang, X.P. Seeking for sterile neutrinos with displaced leptons at the LHC. J. High Energy Phys. 2019, 7, 159. [CrossRef]

166. Das, A.; Dev, P.S.B.; Okada, N. Long-lived TeV-scale right-handed neutrino production at the LHC in gauged $U(1)_{X}$ model. Phys. Lett. B 2019, 799, 135052. [CrossRef]

167. Drewes, M.; Giammanco, A.; Hajer, J.; Lucente, M. New long-lived particle searches in heavy-ion collisions at the LHC. Phys. Rev. D 2020, 101, 055002. [CrossRef]

168. Chiang, C.W.; Cottin, G.; Das, A.; Mandal, S. Displaced heavy neutrinos from $Z^{\prime}$ decays at the LHC. J. High Energy Phys. 2019, 12, 070. [CrossRef]

169. Dib, C.O.; Helo, J.C.; Nayak, M.; Neill, N.A.; Soffer, A.; Zamora-Saa, J. Searching for a sterile neutrino that mixes predominantly with $v_{\tau}$ at $B$ factories. Phys. Rev. D 2020, 101, 093003. [CrossRef]

170. Jones-Pérez, J.; Masias, J.; Ruiz-Álvarez, J.D. Search for Long-Lived Heavy Neutrinos at the LHC with a VBF Trigger. Eur. Phys. J. C 2020, 80, 642. [CrossRef]

171. Barducci, D.; Bertuzzo, E.; Caputo, A.; Hernandez, P.; Mele, B. The see-saw portal at future Higgs Factories. J. High Energy Phys. 2021, 3, 117. [CrossRef]

172. Borsato, M.; Cid Vidal, X.; Tsai, Y.; Vázquez Sierra, C.; Zurita, J.; Alonso-Álvarez, G.; Boyarsky, A.; Brea Rodríguez, A.; Buarque Franzosi, D.; Cacciapaglia, G.; et al. Unleashing the full power of LHCb to probe Stealth New Physics. arXiv 2021, arXiv:hep-ph/2105.12668.

173. Tastet, J.L.; Ruchayskiy, O.; Timiryasov, I. Reinterpreting the ATLAS bounds on heavy neutral leptons in a realistic neutrino oscillation model. J. High Energy Phys. 2021, 12, 182. [CrossRef]

174. Liu, W.; Kulkarni, S.; Deppisch, F.F. Heavy Neutrinos at the FCC-hh in the $U(1)_{B-L}$ Model. arXiv 2022, arXiv:hep-ph/2202.07310.

175. Bray, S.; Lee, J.S.; Pilaftsis, A. Resonant CP violation due to heavy neutrinos at the LHC. Nucl. Phys. B 2007, 786, 95-118. [CrossRef]

176. Tapia, S.; Vidal-Bravo, M.; Zamora-Saa, J. Discovering heavy neutrino oscillations in rare $B_{c}^{ \pm}$meson decays at HL-LHCb. arXiv 2021, arXiv:hep-ph/2109.06027.

177. Cvetič, G.; Kim, C.S.; Zamora-Saá, J. CP violation in the rare Higgs decays via exchange of on-shell almost degenerate Majorana neutrinos, $H \rightarrow v_{k} N_{j} \rightarrow v_{k} \ell^{-} U \bar{D}$ and $H \rightarrow v_{k} N_{j} \rightarrow v_{k} \ell^{+} \bar{U} D$. arXiv 2021, arXiv:hep-ph/2110.08799.

178. Abada, A.; Hati, C.; Marcano, X.; Teixeira, A.M. Interference effects in LNV and LFV semileptonic decays: the Majorana hypothesis. J. High Energy Phys. 2019, 9, 017. [CrossRef]

179. Tumasyan, A.; CMS Collaboration. Search for a right-handed W boson and a heavy neutrino in proton-proton collisions at $\sqrt{s}=13 \mathrm{TeV}$. arXiv 2021, arXiv:hep-ex/2112.03949.

180. Nemevšek, M.; Nesti, F.; Popara, G. Keung-Senjanović process at the LHC: From lepton number violation to displaced vertices to invisible decays. Phys. Rev. D 2018, 97, 115018. [CrossRef]

181. Chauhan, G.; Dev, P.S.B.; Mohapatra, R.N.; Zhang, Y. Perturbativity constraints on $U(1)_{B-L}$ and left-right models and implications for heavy gauge boson searches. J. High Energy Phys. 2019, 1, 208. [CrossRef]

182. Mitra, M.; Ruiz, R.; Scott, D.J.; Spannowsky, M. Neutrino Jets from High-Mass $W_{R}$ Gauge Bosons in TeV-Scale Left-Right Symmetric Models. Phys. Rev. D 2016, 94, 095016. [CrossRef]

183. Ferrari, A.; Collot, J.; Andrieux, M.L.; Belhorma, B.; de Saintignon, P.; Hostachy, J.Y.; Martin, P.; Wielers, M. Sensitivity study for new gauge bosons and right-handed Majorana neutrinos in $p p$ collisions at $s=14-\mathrm{TeV}$. Phys. Rev. D 2000, 62, 013001. [CrossRef]

184. Aaboud, M.; ATLAS Collaboration. Search for heavy Majorana or Dirac neutrinos and right-handed $W$ gauge bosons in final states with two charged leptons and two jets at $\sqrt{s}=13 \mathrm{TeV}$ with the ATLAS detector. J. High Energy Phys. 2019, 1, 016. [CrossRef]

185. Aaboud, M.; ATLAS Collaboration. Search for a right-handed gauge boson decaying into a high-momentum heavy neutrino and a charged lepton in $p p$ collisions with the ATLAS detector at $\sqrt{s}=13 \mathrm{TeV}$. Phys. Lett. B 2019, 798, 134942. [CrossRef]

186. Maiezza, A.; Nemevšek, M.; Nesti, F. Lepton Number Violation in Higgs Decay at LHC. Phys. Rev. Lett. 2015, 115, 081802. [CrossRef]

187. Nemevšek, M.; Nesti, F.; Vasquez, J.C. Majorana Higgses at colliders. J. High Energy Phys. 2017, 4, 114. [CrossRef]

188. Cottin, G.; Helo, J.C.; Hirsch, M. Searches for light sterile neutrinos with multitrack displaced vertices. Phys. Rev. D 2018, 97, 055025. [CrossRef]

189. Cottin, G.; Helo, J.C.; Hirsch, M.; Silva, D. Revisiting the LHC reach in the displaced region of the minimal left-right symmetric model. Phys. Rev. D 2019, 99, 115013. [CrossRef]

190. Langacker, P.; Plumacher, M. Flavor changing effects in theories with a heavy $Z^{\prime}$ boson with family nonuniversal couplings. Phys. Rev. D 2000, 62, 013006. [CrossRef]

191. Langacker, P. The Physics of Heavy Z' Gauge Bosons. Rev. Mod. Phys. 2009, 81, 1199-1228. [CrossRef] 
192. del Aguila, F.; de Blas, J.; Perez-Victoria, M. Electroweak Limits on General New Vector Bosons. J. High Energy Phys. 2010, 9, 033. [CrossRef]

193. de Blas, J.; Lizana, J.M.; Perez-Victoria, M. Combining searches of $Z^{\prime}$ and W' bosons. J. High Energy Phys. 2013, 1, 166. [CrossRef]

194. Accomando, E.; Becciolini, D.; Belyaev, A.; Moretti, S.; Shepherd-Themistocleous, C. $Z^{\prime}$ at the LHC: Interference and Finite Width Effects in Drell-Yan. J. High Energy Phys. 2013, 10, 153. [CrossRef]

195. Jezo, T.; Klasen, M.; Lamprea, D.R.; Lyonnet, F.; Schienbein, I. NLO+NLL limits on $W^{\prime}$ and $Z^{\prime}$ gauge boson masses in general extensions of the Standard Model. J. High Energy Phys. 2014, 12, 092. [CrossRef]

196. Accomando, E.; Coriano, C.; Delle Rose, L.; Fiaschi, J.; Marzo, C.; Moretti, S. $Z^{\prime}$, Higgses and heavy neutrinos in $U(1)^{\prime}$ models: from the LHC to the GUT scale. J. High Energy Phys. 2016, 7, 086. [CrossRef]

197. Deppisch, F.; Kulkarni, S.; Liu, W. Heavy neutrino production via $Z^{\prime}$ at the lifetime frontier. Phys. Rev. D 2019, 100, 035005. [CrossRef]

198. Buras, A.J.; Crivellin, A.; Kirk, F.; Manzari, C.A.; Montull, M. Global analysis of leptophilic Z' bosons. J. High Energy Phys. 2021, 6, 068. [CrossRef]

199. Bossi, F.; Ciafaloni, P. Lepton Flavor Violation at muon-electron colliders. J. High Energy Phys. 2020, 10, 033. [CrossRef]

200. Wetterich, C. Neutrino Masses and the Scale of B-L Violation. Nucl. Phys. B 1981, 187, 343-375. [CrossRef]

201. Buchmuller, W.; Greub, C.; Minkowski, P. Neutrino masses, neutral vector bosons and the scale of B-L breaking. Phys. Lett. B 1991, 267, 395-399. [CrossRef]

202. Emam, W.; Khalil, S. Higgs and Z-prime phenomenology in B-L extension of the standard model at LHC. Eur. Phys. J. C 2007, 52, 625-633. [CrossRef]

203. Basso, L.; Belyaev, A.; Moretti, S.; Shepherd-Themistocleous, C.H. Phenomenology of the minimal B-L extension of the Standard model: $Z^{\prime}$ and neutrinos. Phys. Rev. D 2009, 80, 055030. [CrossRef]

204. Fileviez Perez, P.; Han, T.; Li, T. Testability of Type I Seesaw at the CERN LHC: Revealing the Existence of the B-L Symmetry. Phys. Rev. D 2009, 80, 073015. [CrossRef]

205. Heeck, J. Unbroken B-L symmetry. Phys. Lett. B 2014, 739, 256-262. [CrossRef]

206. Khalil, S. Low scale B-L extension of the Standard Model at the LHC. J. Phys. G 2008, 35, 055001. [CrossRef]

207. Huitu, K.; Khalil, S.; Okada, H.; Rai, S.K. Signatures for right-handed neutrinos at the Large Hadron Collider. Phys. Rev. Lett. 2008, 101, 181802. [CrossRef]

208. Accomando, E.; Delle Rose, L.; Moretti, S.; Olaiya, E.; Shepherd-Themistocleous, C.H. Extra Higgs boson and $Z^{\prime}$ as portals to signatures of heavy neutrinos at the LHC. J. High Energy Phys. 2018, 2, 109. [CrossRef]

209. Dev, P.S.B.; Mohapatra, R.N.; Zhang, Y. Leptogenesis constraints on $B-L$ breaking Higgs boson in TeV scale seesaw models. J. High Energy Phys. 2018, 3, 122. [CrossRef]

210. Davidson, S.; Lacroix, S.; Verdier, P. LHC sensitivity to lepton flavour violating Z boson decays. J. High Energy Phys. 2012, 9, 092. [CrossRef]

211. Abada, A.; Bečirević, D.; Lucente, M.; Sumensari, O. Lepton flavor violating decays of vector quarkonia and of the $Z$ boson. Phys. Rev. D 2015, 91, 113013. [CrossRef]

212. De Romeri, V.; Herrero, M.J.; Marcano, X.; Scarcella, F. Lepton flavor violating Z decays: A promising window to low scale seesaw neutrinos. Phys. Rev. D 2017, 95, 075028. [CrossRef]

213. Herrero, M.J.; Marcano, X.; Morales, R.; Szynkman, A. One-loop effective LFV Z $l_{k} l_{m}$ vertex from heavy neutrinos within the mass insertion approximation. Eur. Phys. J. C 2018, 78, 815. [CrossRef]

214. Langacker, P.; London, D. Mixing Between Ordinary and Exotic Fermions. Phys. Rev. D 1988, 38, 886. [CrossRef]

215. Altmannshofer, W.; Chen, C.Y.; Bhupal Dev, P.S.; Soni, A. Lepton flavor violating Z' explanation of the muon anomalous magnetic moment. Phys. Lett. B 2016, 762, 389-398. [CrossRef]

216. CMS Collaboration. Search for heavy resonances and quantum black holes in $\mathrm{e} \mu, \mathrm{e} \tau$, and $\mu \tau$ final states in proton-proton collisions at $\sqrt{s}=13 \mathrm{TeV}$, CMS-PAS-EXO-19-014. 2021. Available online: http://cds.cern.ch/record/2779023 (accessed on 22 August 2021)

217. Dev, P.S.B.; Rodejohann, W.; Xu, X.J.; Zhang, Y. MUonE sensitivity to new physics explanations of the muon anomalous magnetic moment. J. High Energy Phys. 2020, 5, 053. [CrossRef]

218. Masiero, A.; Paradisi, P.; Passera, M. New physics at the MUonE experiment at CERN. Phys. Rev. D 2020, 102, 075013. [CrossRef]

219. Carloni Calame, C.M.; Passera, M.; Trentadue, L.; Venanzoni, G. A new approach to evaluate the leading hadronic corrections to the muon $g$-2. Phys. Lett. B 2015, 746, 325-329. [CrossRef]

220. Abbiendi, G.; Carloni Calame, C.M.; Marconi, U.; Matteuzzi, C.; Montagna, G.; Nicrosini, O.; Passera, M.; Piccinini, F.; Tenchini, R.; Trentadue, L.; et al. Measuring the leading hadronic contribution to the muon g-2 via $\mu$ e scattering. Eur. Phys. J. C 2017, 77, 139. [CrossRef] 